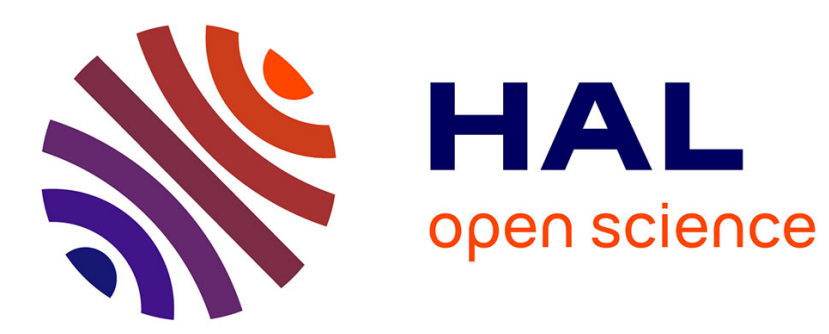

\title{
Implications of vacuum stability constraints on the nonminimal supersymmetric standard model with lepton number violation
}

Marc Chemtob, P.N. Pandita

\section{- To cite this version:}

Marc Chemtob, P.N. Pandita. Implications of vacuum stability constraints on the nonminimal supersymmetric standard model with lepton number violation. Physical Review D, 2007, 76 (9), pp.095019. 10.1103/PhysRevD.76.095019 . cea-02905703

\section{HAL Id: cea-02905703 https://hal-cea.archives-ouvertes.fr/cea-02905703}

Submitted on 23 Jul 2020

HAL is a multi-disciplinary open access archive for the deposit and dissemination of scientific research documents, whether they are published or not. The documents may come from teaching and research institutions in France or abroad, or from public or private research centers.
L'archive ouverte pluridisciplinaire HAL, est destinée au dépôt et à la diffusion de documents scientifiques de niveau recherche, publiés ou non, émanant des établissements d'enseignement et de recherche français ou étrangers, des laboratoires publics ou privés. 


\title{
Implications of vacuum stability constraints on the nonminimal supersymmetric standard model with lepton number violation
}

\author{
M. Chemtob* \\ Service de Physique Théorique, CEA-Saclay F-91191 Gif-sur-Yvette Cedex France \\ P.N. Pandita ${ }^{\dagger}$ \\ Department of Physics, North Eastern Hill University, Shillong 793 022, India
}

\begin{abstract}
We carry out a detailed analysis of the scalar sector of the nonminimal supersymmetric standard model with lepton number violation, and study the constraints imposed on it by the stability of the electroweak symmetry breaking vacuum. The model contains a trilinear lepton mumber violating term in the superpotential together with the associated supersymmetry breaking interactions which can give rise to neutrino masses. We evaluate the mass matrices for the various boson and fermion modes and then discuss the effect that the lepton number violating interactions have on the mass spectra using a phenomenological precription to implement the experimental constraints on the light neutrinos mass matrix. We also discuss qualitatively the conditions on the lepton number violating parameters set by the unbounded from below directions, and from the absence of the charge and color breaking minima in this model.

PACS numbers: 12.60.Jv, 14.60.Ly, 11.30.Fs
\end{abstract}

*Electronic address: marc. chemtob@cea.fr

${ }^{\dagger}$ Electronic address: ppandita@nehu.ac.in 


\section{INTRODUCTION}

There are suggestive hints of lepton number violation in the observation of neutrino flavor oscillations [1]. The link between the new physics beyond the Standard Model (SM) and lepton number violating interactions has so far been realized in two main theoretical frameworks. The first option, the see-saw mechanism [2], uses physics at the grand unified scale, with lepton number violation represented by the non-renormalizable interaction of dimension $5, \mathcal{L}_{\text {eff }}=\frac{1}{\Lambda}\left(L H_{u}\right)\left(L H_{u}\right)$, where $L$ and $H_{u}$ are the lepton and Higgs doublets, respectively. The second option involves minimal supersymmetric standard model (MSSM) with broken (generalized) lepton parity, and uses bilinear and/or trilinear lepton number violating couplings in the superpotential, with lepton number violation occurring at the weak scale [3].

Recently, we have pointed out [4] that in the context of low energy supersymmetry there is an attractive option of generating neutrino masses in the nonminimal supersymmetric standard model (NMSSM) with trilinear lepton number violation. Unlike the minimal supersymmetric standard model (MSSM), the superpotential of the NMSSM is scale invariant. What is perhaps even more interesting is the presence of an additional lepton number violating trilinear coupling in the superpotential of NMSSM which has no analog in the MSSM. In the NMSSM with lepton number violation we have a situation which is different from the one that arises in the see-saw mechanism or the bilinear lepton number violation in MSSM in that no dimensionful mass parameters (large or small) are introduced.

There are several reasons for studying the nonminimal supersymmetric standard model. First, and foremost, it is the simplest supersymmetric extension of the Standard Model in which the electroweak scale originates from the supersymmetry breaking scale only. Second, and as mentioned above, this model breaks lepton number and $R$-parity explicitly through a unique trilinear superpotential term $[5,6]$. Third, the model can successfully explain the

pattern of neutrino masses with normal type of hierarchy [4] without invoking either a large or a small mass parameter. And last, but not the least, its enlarged Higgs sector may help in relaxing the fine-tuning and little hierarchy problems of the MSSM [7], thereby opening new perspectives for the Higgs boson searches at the next generation of high energy colliders.

In addition to the familiar Yukawa interaction superpotential for the three generations of quark and lepton superfields, and for the Higgs bosons superfields, $H_{u}, H_{d}$, the model 
couples the set of down type Higgs boson and sleptons, $L_{A}=\left(H_{d}, L_{i}\right)(A=d, i ; i=1,2,3)$, with the Higgs singlet superfield $S$ through the superpotential $W=\tilde{\lambda}_{A} L_{A} H_{u} S-\frac{\kappa}{3} S^{3}$, where $\tilde{\lambda}_{A}=\left(\lambda, \quad \tilde{\lambda}_{i}\right)$ and $\kappa$ are dimensionless parameters. The familiar soft supersymmetry breaking trilinear scalar couplings and mass terms introduce the dimensional parameters $A_{\tilde{\lambda}_{A}}=\left(A_{\lambda}, A_{\tilde{\lambda}_{i}}\right), A_{\kappa}$, and $m_{\tilde{L}, A B}^{2}, m_{S}^{2}$. Whereas the fundamental parameters in the action are $\tilde{\lambda}_{A}, A_{\tilde{\lambda}_{A}}, m_{\tilde{L}, A B}^{2}$, the vacuum solution is specified by the dynamical parameters corresponding to the vacuum expectation values (VEVs) of the electrically neutral scalar fields at the electroweak scale, $v_{u}=<H_{u}>, v_{A}=\left(v_{d}=<H_{d}>, v_{i}=<\tilde{\nu}_{i}>\right)$, which include the sneutrinos together with the neutral components of the Higgs bosons. We use a covariant notation for the down-type Higgs boson and slepton superfields, and their couplings, in order to illustrate the invariance of observables under the $S U(4)$ group of transformations acting on the $4 \mathrm{D}$ column vectors $L_{A}$ and $\tilde{\lambda}_{A}, A_{\tilde{\lambda}_{A}}$, and the matrix $m_{\tilde{L}, A B}^{2}$. Strong correlations usually exist between the fundamental and the dynamical parameters. Thus, the experimental observations for the light neutrino mass matrix tightly constrain certain combinations of the parameters which characterize the inequivalent field directions distinguishing the down-type Higgs boson from the sleptons, and hence the interactions which conserve lepton number from those which violate it. One may hope that imposing the necessary restrictions on the misalignment type parameters would still leave some freedom in the parameter space so as to allow the lepton number violating interactions to significantly affect the physics of the NMSSM. There is a close analogy here with the situation that obtains in the MSSM with R-parity violation [8-15].

At the tree level, the size of the neutrino Majorana mass matrix is knownn to be controlled by deviations from the $\tilde{\lambda}_{A} \propto v_{A}$ alignment, such that the predicted single non-vanishing neutrino mass is proportional to $\sum_{i} \tilde{\lambda}_{i}^{\prime 2}$, where the effective misalignment parameters are defined by the linear combinations $\tilde{\lambda}_{i}^{\prime} \equiv \tilde{\lambda}_{i}-\lambda v_{i} / v_{d}$. We have previously shown [4] that the suitably constrained NMSSM predicts an acceptable neutrino mass matrix of a normal hierarchy type so that the tentative limits on the heaviest neutrino mass, $m_{\nu_{\tau}}<(10 \mathrm{MeV}-1 \mathrm{eV})$, set bounds on the effective parameters given by $\tilde{\lambda}_{i}^{\prime}<\left(3.10^{-2}-10^{-5}\right)$. Bounds of similar type were found for the couplings $\tilde{\lambda}_{i}$ in the basis $v_{i}=0$ in an attempt to fit an extended version of the model to the neutrino mass differences and mixing angles [16]. It has also been observed that ratios as large as $\tilde{\lambda}_{i} / \lambda \sim 10^{-1}$ might be allowed at the price of fine tuning the NMSSM parameters [17]. As far as one-loop contributions to the neutrino mass 
matrix is concerned, the situation is more complex due to the larger number of unknown parameters and the presence of several sources of contributions arising in perturbation theory. Thus, to supress the contributions from the one-loop amplitude involving the exchange of scalars and gauginos, it is necessary to impose strong constraints on the effective parameters, $B_{i}^{\prime}=B_{i}-B v_{i} / v_{d}\left[B_{A}=\left(B, B_{i}\right) \equiv\left(A_{\tilde{\lambda}_{i}} \tilde{\lambda}_{i}, A_{\lambda} \lambda\right)\right]$ controlled by the $B_{A} \propto v_{A}$ alignment.

Supersymmetric models, including the nonminimal supersymmetric standard model, contain a large number of scalar fields, such as the scalar partners of quarks and leptons, which are not present in the Standard Model. As a result, the scalar potential of supersymmetric models is fairly complicated. Such a potential can have minima which could lead to the breakdown of color or electric charge conservation through the vacuum expectation values of squark and slepton fields. The condition that the global minimum of the theory does not violate color or electromagnetic gauge invariance provides useful constraints on the parameter space of the underlying supersymmetric model, as has been well illustrated in the context of the minimal supersymmetric standard model [18-22] and its extended version with broken R parity symmetry [23, 24]. Significant bounds can be obtained not only on the value of the trilinear soft supersymmetry breaking parameters, but also on the values of the bilinear soft scalar supersymmetry breaking parameter, as well as scalar and gaugino masses, respectively.

The possibility that one can reproduce the lepton number violating observables without imposing strong restrictions on the coupling constants $\tilde{\lambda}_{i}$ motivates us to examine how the scalar sector of the NMSSM is affected by treating $\tilde{\lambda}_{i}$ as free parameters. One may expect interesting implications regarding the stability of the regular vacuum solution and the properties of the neutral and charged scalars. While performing the calculations within a field basis independent formalism [8-10, 12-15] is highly desirable, this is very tedious and is not particularly illuminating. It requires an unusually large amount of effort which is not warranted at the present stage of our study. We, thus, choose to pursue the calculations within a basis dependent formalism. In principle, there is a choice of basis for the neutrino superfields in which the sneutrino VEVs can be set to zero at the expense of transferring through a superfield redefinition the couplings $L_{i} H_{u} S$ to the trilinear lepton number violating couplings of the matter superfields. Although the calculations are greatly simplified in the basis $v_{i}=0$, there is a risk that this choice biases the parameter space exploration, as has clearly been pointed out in the above discussion regarding the neutrino mass matrix. In 
fact, in the basis choice $v_{i}=0$, the couplings $\tilde{\lambda}_{i}$ are so strongly suppressed that the issue of establishing any observable effect on the scalar sector would be closed from the very start.

In the present work, we shall adopt the view that the lepton number violation phenomenology allows the couplings $\tilde{\lambda}_{i}$ to cover a wide range of variation, $0 \leq\left|\tilde{\lambda}_{i} / \lambda\right| \leq 1$. Thus, we treat all the $\tilde{\lambda}_{A}$ on the same footing, while requiring the soft and dynamical parameters to satisfy the near alignment properties, $v_{A} \propto B_{A}$ and $v_{A} \propto \tilde{\lambda}_{A}$, in agreement with the observations. Following a phenomenological approach, we treat $\tilde{\lambda}_{i}$ as free parameters, and determine $v_{i}$ and $B_{i}$ by restricting the linear combinations $\tilde{\lambda}_{i}^{\prime}=\tilde{\lambda}_{i}-\lambda v_{i} / v_{d}$ and $B_{i}^{\prime}=B_{i}-B v_{i} / v_{d}$. Since $v_{d}, v_{i}$ are determined by minimizing the scalar potential, this procedure implies a fine tuning. However, one can use symmetries to impose in a natural way a preferred direction in the $L_{A}$ field space along which the various parameters get aligned. While the horizontal flavor discrete symmetries $[25,26]$ indeed have the ability to enforce an approximate $\tilde{\lambda}_{A} \propto v_{A}$ alignment, this property is robust only in the case of hierarchically small ratios, $\frac{\tilde{\lambda}_{i}}{\lambda} \sim \frac{v_{i}}{v_{d}}$, which is not exactly what we want. By contrast, the supergravity inspired models of the type discussed by Nilles and Polonsky [27], in which the $\tilde{\lambda}_{A} L_{A} H_{u} S$ couplings arise from non-renormalizable interactions controlled by a spontaneously broken $U(1)_{R}$ type symmetry, are compatible with all the $\tilde{\lambda}_{A}$ having the same size. The alignment is realized dynamically via universal type boundary conditions on the soft parameters, and remains typically stable under the renormalization group [27, 28]. Our main purpose is to study the impact of the lepton number violation on the electroweak symmetry breaking vacuum of the nonminimal supersymmetric standard model consistent with the experimental measurements for the light neutrinos. In the first stage, we discuss its stability by testing for the occurrence of tachyons (negative eigenvalues of the squared mass matrices) for the charged and neutral scalar modes. In the second stage, we analyze the dangerous directions in the scalar field space along which the scalar potential is unbounded from below (UFB) or develops deeper charge and/or color breaking (CCB) minima. Distinct methods must be used at these two stages, which both involve the non-supersymmetric couplings in an essential way.

This paper is organized as follows. In Section II we describe the nonminimal supersymmetric standard model with lepton number violation, and set up our notation and conventions. Complementary definitions and results are summarized in the Appendix A. In Section III we discuss the conditions on the superpotential couplings and supersymmetry 
breaking couplings required to exclude the tachyon scalar modes for the regular vacuum. In Section IV we explore the conditions on the superpotential and supersymemtry breaking couplings implied by the lifting of UFB directions, and the removal of CCB minima. In Section V we summarize our main conclusions.

\section{THE NMSSM WITH BARYON AND LEPTON NUMBER VIOLATION}

In this section we summarize the basic features of the superpotential and the associated soft breaking terms for the nonminimal supersymmetric standard model with baryon and lepton number violation, and establish our notation and conventions. Some of the details are further discussed in the Appendix A.

\section{A. Superpotential and soft breaking terms}

The superpotential of NMSSM with baryon and lepton number violation is characterized by the scale invariant supepotential

$$
W=W_{R P C}+W_{R P V}
$$

where $W_{R P C}$ and $W_{R P V}$ are the baryon and lepton number (and $R$-parity) conserving, and baryon and lepton number (and $R$-parity) violating contributions, respectively to the superpotential [4]. These contributions are written down explicitly in Appendix A. In the absence of lepton number conservation, there is no distinction between the down-type Higgs $\left(H_{d}\right)$ and the lepton superfields $\left(L_{i}\right)$ as they transform identically under the SM gauge group. We can, therefore, employ a four vector notation for the down type Higgs and the lepton superfields, and the Yukawa couplings

$$
\begin{aligned}
& L_{A}=\left(H_{d}, L_{i}\right), \\
& \tilde{\lambda}_{A}=\left(\lambda, \tilde{\lambda}_{i}\right),[A=d, i ; i=1,2,3],
\end{aligned}
$$

which is given in detail in Eq.(A.4) of Appendix A. The quark and lepton generation indices are denoted by the letters $i, j, \cdots$, and the trilinear couplings obey the antisymemtry property, $\lambda_{A B k}=-\lambda_{B A k}$. In this notation we can write the complete superpotential of 
NMSSM with baryon and lepton number violation as

$$
\begin{aligned}
W & =\lambda_{j k}^{u} H_{u} Q_{j} U_{k}^{c}+\frac{1}{2} \lambda_{A B k} L_{A} L_{B} E_{k}^{c}+\lambda_{A j k}^{\prime} L_{A} Q_{j} D_{k}^{c}+\frac{1}{2} \lambda_{i j k}^{\prime \prime} U_{i}^{c} D_{j}^{c} D_{k}^{c} \\
& +\tilde{\lambda}_{A} L_{A} H_{u} S-\frac{\kappa}{3} S^{3} .
\end{aligned}
$$

The supersymmetric contribution to the Lagrangian of NMSSM with baryon and lepton number violation generated by the superpotential (II.3) can be obtained by a standard procedure. To this we must add the supersymmetric contribution from the $D$-terms generated by the gauge interactions. In addition to the supersymmetric part, the Lagrangian consists of soft supersymmetry breaking terms, which include soft trilinear scalar couplings and soft masses for all scalars and gauginos, respectively. The part of the soft supersymmetry breaking scalar potential of NMSSM containing trilinear scalar couplings can be written as (note that it is $-V$ that occurs in the Lagrangian)

$$
\begin{aligned}
V^{\mathrm{soft}}(\text { trilinear })= & V_{R P C}^{\mathrm{soft}}(\text { trilinear })+V_{R P V}^{\mathrm{soft}}(\text { trilinear }), \\
= & -\frac{1}{2} A_{A B k}^{\lambda} \lambda_{A B k} \tilde{L}_{A} \tilde{L}_{B} \tilde{E}_{k}^{c}-A_{A j k}^{\lambda^{\prime}} \lambda_{A j k}^{\prime} \tilde{L}_{A} \tilde{Q}_{j} \tilde{D}_{k}^{c}-A_{j k}^{u} \lambda_{j k}^{u} H_{u} \tilde{Q}_{j} \tilde{U}_{k}^{c} \\
& -\frac{1}{2} A_{i j k}^{\lambda^{\prime \prime}} \lambda_{i j k}^{\prime \prime} \tilde{U}_{i}^{c} \tilde{D}_{j}^{c} \tilde{D}_{k}^{c}-A_{\tilde{\lambda}_{A}} \tilde{\lambda}_{A} \tilde{L}_{A} H_{u} S-\frac{A_{\kappa} \kappa}{3} S^{3}+H . c .,
\end{aligned}
$$

and the part containing the bilinear mass terms for the scalars and gauginos can be written as

$$
V^{\mathrm{soft}}(\text { mass })=V_{R P C}^{\mathrm{soft}}(\text { mass })+V_{R P V}^{\mathrm{soft}}(\text { mass }),
$$

with the lepton number conserving RPC terms given by

$$
\begin{aligned}
V_{R P C}^{\mathrm{Soft}}(\mathrm{mass}) & =m_{\tilde{Q}_{i}}^{2}\left|\tilde{Q}_{i}\right|^{2}+m_{\tilde{U}_{i}^{c}}^{2}\left|\tilde{U}_{i}^{c}\right|^{2}+m_{\tilde{D}_{i}^{c}}^{2}\left|\tilde{D}_{i}^{c}\right|^{2}+m_{\tilde{L}_{i}}^{2}\left|\tilde{L}_{i}\right|^{2}+m_{\tilde{E}_{i}^{c}}^{2}\left|\tilde{E}_{i}^{c}\right|^{2}, \\
& +m_{H_{u}}^{2}\left|H_{u}\right|^{2}+m_{H_{d}}^{2}\left|H_{d}\right|^{2}+m_{S}^{2}|S|^{2} \\
& -\left(\sum_{a=3,2,1} \frac{1}{2} M_{a} \tilde{\lambda}_{a} \tilde{\lambda}_{a}+\text { H. c. }\right)
\end{aligned}
$$

and the lepton number violating terms given by

$$
V_{R P V}^{\mathrm{soft}}(\mathrm{mass})=m_{H_{d} \tilde{L}_{i}}^{2} H_{d}^{\dagger} \tilde{L}_{i}+\mu_{H_{d} H_{u}}^{2} H_{d} H_{u}+\mu_{\tilde{L}_{i} H_{u}}^{2} \tilde{L}_{i} H_{u}+\text { H.c. }
$$

We have used the convention in which the repeated indices are implicitly summed over and have suppressed the $S U(3)$ color quantum numbers of the quark and squark fields by setting, for instance, $\tilde{Q}_{i}^{\alpha}=\left(\tilde{U}_{i}^{\alpha}, \tilde{D}_{i}^{\alpha}\right) \rightarrow \tilde{Q}_{i}=\left(\tilde{U}_{i}, \tilde{D}_{i}\right)$. Although we have represented the 
fields mass mixing by the general terms, $m_{\tilde{L}, i j}^{2} \tilde{L}_{i}^{\dagger} \tilde{L}_{j}$ and $m_{\tilde{L}, A B}^{2} \tilde{L}_{A}^{\dagger} \tilde{L}_{B}$, for the sleptons and for the down Higgs boson and sleptons, we neglect thoughout the present work the intergenerational mixing of sleptons by assuming that $\tilde{L}_{i}$ are mass basis fields with $m_{\tilde{L}, i j}^{2}=m_{\tilde{L}_{i}}^{2} \delta_{i j}$ while retaining the off-diagonal mass parameters, $m_{H_{d} \tilde{L}_{i}}^{2} \equiv m_{d i}^{2} \neq 0$. Since supersymmetry breaking respects the SM gauge symmetry, we must assign the same soft mass parameters for the two members of electroweak doublet fields, as is explicitly done for the expression of the slepton mass terms, viz. $m_{\tilde{L}_{i}}^{2}\left|\tilde{L}_{i}\right|^{2}=m_{\tilde{L}_{i}}^{2}\left(\left|\tilde{\nu}_{i}\right|^{2}+\left|\tilde{e}_{i}\right|^{2}\right)$. The choice of the sign of $M_{2}$ in the chargino mass matrix conforms with the convention most often used in the literature. We have also included above, for generality, the holomorphic mass mixing terms with the parameters $\mu_{A u}^{2}=\left(\mu_{H_{d} H_{u}}^{2} \equiv \mu_{d u}^{2}, \mu_{\tilde{L}_{i} H_{u}}^{2} \equiv \mu_{i u}^{2}\right)$, although the soft bilinear operators $\mu_{A u}^{2} \tilde{L}_{A} H_{u}$ are, in principle, disallowed, since the same discrete symmetry that forbids the bilinear superpotential terms $\mu_{A} L_{A} H_{u}$ should also forbid the bilinear mass mixing operators. Mass mixing terms of same structure would still arise as effective contributions through the soft trilinear operators, $-A_{\tilde{\lambda}_{A}} \tilde{\lambda}_{A}<S>\tilde{L}_{A} H_{u}$, with $\mu_{A u}^{2}=-A_{\tilde{\lambda}_{A}} \tilde{\lambda}_{A}<S>$.

The scalar potential receives contributions from the supersymmetric as well as the soft supersymmetry breaking interactions involving the electrically neutral and charged complex scalar fields. The supersymmetric contribution arises from the superpotential (II.3) (the $F$-terms), and from the $D$-terms. The Higgs boson and slepton $F$-term contributions to the scalar potential can be written as

$$
\left.V_{F} \text { (Higgs, sleptons }\right)=\left|W_{H_{u}^{0}}\right|^{2}+\left|W_{H_{u}^{+}}\right|^{2}+\left|W_{S}\right|^{2}+\left|W_{\tilde{\nu}_{A}}\right|^{2}+\left|W_{\tilde{e}_{A}}\right|^{2}+\left|W_{\tilde{e}_{i}^{c}}\right|^{2},
$$

where

$$
\begin{aligned}
W_{H_{u}^{0}} & =-\lambda_{j k}^{u} u_{j} u_{k}^{c}+\tilde{\lambda}_{A} v_{A} x, \quad W_{H_{u}^{+}}=\lambda_{j k}^{u} d_{j} u_{k}^{c}-\tilde{\lambda}_{A} e_{A} x, \\
W_{S} & =\tilde{\lambda}_{A} v_{A} v_{u}-\tilde{\lambda}_{A} e_{A} v_{+}-\kappa x^{2}, \\
W_{\tilde{\nu}_{A}} & =\lambda_{A B k} e_{B} e_{k}^{c}+\lambda_{A j k}^{\prime} d_{j} d_{k}^{c}+\tilde{\lambda}_{A} v_{u} x, \\
W_{\tilde{e}_{A}} & =-\lambda_{A j k}^{\prime} u_{j} d_{k}^{c}-\lambda_{B A k} v_{B} e_{k}^{c}-\tilde{\lambda}_{A} v_{+} x, \\
W_{\tilde{e}_{i}^{c}} & =\lambda_{A B i} v_{A} e_{B},
\end{aligned}
$$

with various vacuum expectation values denoted by

$$
\begin{aligned}
& v_{A}=\left(v_{d}=<H_{d}^{0}>, v_{i}=<\tilde{\nu}_{i}>\right), e_{A}=\left(v_{-}=<H_{d}^{-}>, e_{i}=<\tilde{e}_{i}>\right), \\
& e_{i}^{c}=<\tilde{e}_{i}^{c}>, v_{+}=<H_{u}^{+}>, v_{u}=<H_{u}^{0}>.
\end{aligned}
$$


The $F$-term contribution of the squarks to the scalar potential can be written as

$$
V_{F}(\text { squarks })=\left|W_{u_{i}}\right|^{2}+\left|W_{d_{i}}\right|^{2}+\left|W_{u_{i}^{c}}\right|^{2}+\left|W_{d_{i}^{c}}\right|^{2}
$$

where

$$
\begin{aligned}
& W_{u_{i}}=-\lambda_{i k}^{u} v_{u} u_{k}^{c}-\lambda_{A i k}^{\prime} e_{A} d_{k}^{c}, \quad W_{d_{i}}=\lambda_{i k}^{u} v_{+} u_{k}^{c}+\lambda_{A i k}^{\prime} v_{A} d_{k}^{c}, \\
& W_{u_{i}^{c}}=\lambda_{j i}^{u}\left(v_{+} d_{j}-v_{u} u_{j}\right)+\frac{1}{2} \lambda_{i j k}^{\prime \prime} d_{j}^{c} d_{k}^{c}, W_{d_{i}^{c}}=\lambda_{A j i}^{\prime}\left(v_{A} d_{j}-e_{A} u_{j}\right),
\end{aligned}
$$

with the vacuum expectation values of the squarks defined by

$$
u_{i}=<\tilde{U}_{i}>, d_{i}=<\tilde{D}_{i}>, u_{i}^{c}=<\tilde{U}_{i}^{c}>, d_{i}^{c}=<\tilde{D}_{i}^{c}>
$$

The $D$-term contributions to the scalar potential can be written as

$$
V_{D}=V_{D}^{U(1)}+V_{D}^{S U(2)}+V_{D}^{S U(3)}
$$

with

$$
\begin{aligned}
V_{D}^{U(1)}= & \frac{g_{1}^{2}}{8}\left(-\left|v_{d}\right|^{2}-\left|v_{-}\right|^{2}+\left|v_{u}\right|^{2}+\left|v_{+}\right|^{2}+\frac{1}{3}\left|u_{i}\right|^{2}+\frac{1}{3}\left|d_{i}\right|^{2}\right. \\
& \left.-\frac{4}{3}\left|u_{i}^{c}\right|^{2}+\frac{2}{3}\left|d_{i}^{c}\right|^{2}-\left|v_{i}\right|^{2}-\left|e_{i}\right|^{2}+2\left|e_{i}^{c}\right|^{2}\right)^{2}, \\
V_{D}^{S U(2)}= & \frac{g_{2}^{2}}{8}\left[\left(\left|v_{d}\right|^{2}-\left|v_{-}\right|^{2}+\left|v_{+}\right|^{2}-\left|v_{u}\right|^{2}+\left|v_{i}\right|^{2}-\left|e_{i}\right|^{2}+\left|u_{i}\right|^{2}-\left|d_{i}\right|^{2}\right)^{2}\right. \\
& \left.+4\left|v_{d}^{\star} v_{-}+v_{i}^{\star} e_{i}+v_{+}^{\star} v_{u}+u_{i}^{\star} d_{i}\right|^{2}\right], \\
V_{D}^{S U(3)}= & \frac{g_{3}^{2}}{6}\left(\left|u_{i}\right|^{2}+\left|d_{i}\right|^{2}-\left|u_{i}^{c}\right|^{2}-\left|d_{i}^{c}\right|^{2}\right)^{2} .
\end{aligned}
$$

The complete soft supersymmetry breaking scalar potential is given by

$$
\begin{aligned}
V^{\text {soft }}= & m_{\tilde{Q}_{i}}^{2}\left(\left|u_{i}\right|^{2}+\left|d_{i}\right|^{2}\right)+m_{\tilde{U}_{i}^{c}}^{2}\left|u_{i}^{c}\right|^{2}+m_{\tilde{D}_{i}^{c}}^{2}\left|d_{i}^{c}\right|^{2}+m_{\tilde{L}_{i}}^{2}\left(\left|v_{i}\right|^{2}+\left|e_{i}\right|^{2}\right) \\
& +m_{\tilde{E}_{i}^{c}}^{2}\left|e_{i}^{c}\right|^{2}+m_{H_{d}}^{2}\left(\left|v_{d}\right|^{2}+\left|v_{-}\right|^{2}\right)+m_{H_{u}}^{2}\left(\left|v_{u}\right|^{2}+\left|v_{+}\right|^{2}\right)+m_{S}^{2}|x|^{2} \\
& +\left[\mu_{A u}^{2}\left(v_{A} v_{u}-e_{A} v_{+}\right)+m_{H_{d} \tilde{L}_{i}}^{2}\left(v_{d} v_{i}^{\star}+v_{-} e_{i}^{\star}\right)+H . c .\right] \\
& +\left[-A_{\tilde{\lambda}_{A}} \tilde{\lambda}_{A}\left(v_{A} v_{u}-e_{A} v_{+}\right) x-\frac{1}{3} A_{\kappa} \kappa x^{3}+A_{j k}^{u} \lambda_{j k}^{u}\left(u_{j} v_{u}-d_{j} v_{+}\right) u_{k}^{c}-A_{A B k}^{\lambda} \lambda_{A B k} v_{A} e_{B} e_{k}^{c}\right. \\
& \left.-A_{A j k}^{\lambda^{\prime}} \lambda_{A j k}^{\prime}\left(v_{A} d_{j}-e_{A} u_{j}\right) d_{k}^{c}-\frac{1}{2} A_{i j k}^{\lambda^{\prime \prime}} \lambda_{i j k}^{\prime \prime} u_{i}^{c} d_{j}^{c} d_{k}^{c}+H . c .\right]
\end{aligned}
$$

We recall our convention of summing over the repeated indices for the (suppressed) color indices and for the squark and slepton generation labels, $i, j, k, \cdots=1,2,3$. Assuming that 
the squark fields point in a fixed direction in the color space, no summations over the color indices would be present in the above formulae. Furthermore, assuming that the VEVs for the squarks and sleptons of different generations affect the scalar potential independently of each other, allows one to disregard the summations over the generation labels.

In order to discuss NMSSM in a somewhat realistic manner, it is necessary to include the one-loop contributions to the scalar potential. We shall make the usual assumption [29, 30] that the scalar potential is dominated by the top quark and squark modes whose explicit contributions are given by

$$
V_{\text {loop }}=\frac{3}{32 \pi^{2}}\left[\sum_{i=1,2} m_{\tilde{t}_{i}}^{4}\left(\ln \frac{m_{\tilde{t}_{i}}^{2}}{Q^{2}}-\frac{3}{2}\right)-2 \bar{m}_{t}^{4}\left(\ln \frac{\bar{m}_{t}^{2}}{Q^{2}}-\frac{3}{2}\right)\right],
$$

where $\bar{m}_{t}=\lambda_{t} v_{u}$ is the top quark mass and $m_{\tilde{t}_{i}}^{2},(i=1,2)$ denote the squared masses of the top squarks. We do not discuss this point any further, as detailed discussions can be found in Refs. [29-32].

\section{B. Symmetry Constraints on the Parameter Space and Choice of Free Parameters}

The scalar sector involves the scalar field components of the doublet and singlet Higgs boson superfields, and the lepton and quark superfields. The parameter space of the NMSSM with lepton number violation consist of the gauge couplings $g_{a}(a=3,2,1)$, the supersymmetric couplings $\lambda, \kappa, \lambda_{j k}^{u}, \lambda_{j k}^{d}, \lambda_{j k}^{e}, \tilde{\lambda}_{i}, \lambda_{i j k}, \lambda_{i j k}^{\prime}$, the soft supersymmetry breaking couplings $A_{\tilde{\lambda}_{A}} \tilde{\lambda}_{A}, A_{\kappa} \kappa, A_{A B k}^{\lambda} \lambda_{A B k}, A_{A j k}^{\lambda^{\prime}} \lambda^{\prime}{ }_{A j k}, A_{j k}^{u} \lambda_{j k}^{u}$, and the soft supersymmetry breaking mass parameters, $m_{\tilde{Q}_{i}}^{2}, m_{\tilde{U}_{i}^{c}}^{2}, m_{\tilde{D}_{i}^{c}}^{2}, m_{H_{d}}^{2}, m_{H_{u}}^{2}, m_{\tilde{L}_{i}}^{2}, m_{S}^{2}, m_{\tilde{E}_{i}^{c}}^{2}, m_{H_{d} \tilde{L}_{i}}^{2}, \mu_{A u}^{2}$. We summarize below some useful definitions and abbreviations used in this paper:

$$
\begin{aligned}
& m_{W}^{2}=\frac{g_{2}^{2}}{2}\left(v_{u}^{2}+\hat{v}_{d}^{2}\right), m_{Z}^{2}=\frac{g_{1}^{2}+g_{2}^{2}}{2}\left(v_{u}^{2}+v_{d}^{2}+v_{i}^{2}\right), \\
& \tan \beta=\frac{v_{u}}{v_{d}}, G_{ \pm}^{2}=\frac{g_{1}^{2} \pm g_{2}^{2}}{8}, \quad v^{2}=v_{u}^{2}+\hat{v}_{d}^{2}, \quad \hat{v}_{d}^{2}=v_{d}^{2}+v_{i}^{2} .
\end{aligned}
$$

The electroweak symmetry breaking scale has a numerical value $v=\frac{\sqrt{2} m_{W}}{g_{2}} \simeq 174 \mathrm{GeV}$.

Without loss of generality, the parameterization of the NMSSM can be simplified by using the symmetries of the action and the independence of observables under phase redefinitions of the fields. The invariance of the Lagrangian under the $S U(2)_{L} \times U(1)_{Y}$ gauge symmetry allows eliminating four real field degrees of freedom, independently of the structure of the Yukawa couplings. We choose the convention where the VEVs of the up-type Higgs boson 
electroweak doublet are set as $v_{+} \equiv<H_{u}^{+}>=0$ and $v_{u} \equiv<H_{u}^{0}>\in R^{+}$. With the choice $v_{+}=0$, the minimization with respect to the field $H_{u}^{+}$becomes trivial. Next, using the scalar potential invariance under phase redefinitions of the fields $S, H_{d}, \tilde{U}^{c}, \tilde{Q}$ and the pair of fields $H_{d}^{-}, \tilde{D}^{c}$, one can make the following choice [33] for various parameters

$$
\kappa A_{\kappa} \in R^{+}, \lambda A_{\lambda} \in R^{+}, A^{u} \lambda_{u} \in R^{+}, u_{i} \in R^{+}, d_{i} \in R^{+} .
$$

For completeness, we also observe that in the presence of lepton number violation the NMSSM still satisfies a Peccei-Quinn symmetry in the limit $\kappa \rightarrow 0$ and a $R$ type symmetry in the limit $A_{\lambda} \rightarrow 0, A_{\tilde{\lambda}_{i}} \rightarrow 0$, where the $U(1)_{P Q}$ and $U(1)_{R}$ groups are defined by the assignment of charges for $\left(Q, U^{c}, D^{c}, L, E^{c}, H_{d}, H_{u}, S\right), \quad Q_{P Q}=(-1,0,2,-1,2,-1,1,0), Q_{R}=$ $\frac{1}{3}(3,0,2,1,4,1,3,2)$. In the limit $x \rightarrow \infty$, with $\tilde{\lambda}_{A} x=-\mu_{A}, \kappa x, A_{\tilde{\lambda}_{A}} \tilde{\lambda}_{A} x=-B_{A} \mu_{A}$ fixed, the physical observables must reduce to those of the MSSM with bilinear $R$-parity violation [24].

In order to obtain significant contributions to the scalar sector observables from the lepton number violating interactions, some subset of the parameters $\tilde{\lambda}_{i}, A_{\tilde{\lambda}_{i}}, m_{H_{d} \tilde{L}_{i}}^{2}, v_{i}$ should assume large enough values. Whether this can be achieved while imposing at the same time highly suppressed contributions to the neutrino mass matrix is closely related to the formal symmetry under the $S U(4)$ group symmetry in the field space $L_{A}=\left(H_{d}, L_{i}\right)$. The freedom with respect to the choice of $L_{A}$ field basis entails that physical observables can only depend on the $S U(4)$ invariant combinations of the parameters, $\tilde{\lambda}_{A}, B_{A}=A_{\tilde{\lambda}_{A}} \tilde{\lambda}_{A}, m_{\tilde{L}, A B}^{2}, v_{A}$, transforming as vectors or tensors. The physical observables can only depend on the singlet combinations. At the quantum level, the field basis independence holds only after summing all contributions at a given order of the perturbation theory. The basis independent formalism can be developed along similar lines as for the MSSM with R-parity violation [8-10]. Since $H_{d}$ is distinguished from $L_{i}$ by not having lepton number, it follows that the lepton number violating observables can only depend on the invariants of the angle type characterizing the inequivalent directions assigned to $H_{d}$. Thus, the tree level contributions to the neutrino mass matrix involve only the misalignment parameter $|\tilde{\lambda} \wedge v|^{2}=\frac{1}{2} \sum_{A, B}(\tilde{\lambda} \wedge v)_{A B}^{2} \equiv \frac{1}{2} \sum_{A, B}\left(\tilde{\lambda}_{A} v_{B}-\tilde{\lambda}_{B} v_{A}\right)^{2}$, while the one-loop contributions from the scalar-neutralino exchange Feynman diagrams, for instance, involve the invariant misalignment parameters, $|B \wedge v|$ and $|B \wedge \tilde{\lambda}|$. By contrast, the lepton number conserving observables can also depend on the scalar products $\hat{v}_{d}^{2}=\sum_{A} v_{A}^{2}, v \cdot B=\sum_{A} v_{A} B_{A}$, and 
$\tilde{\lambda}^{T} m_{\tilde{L}}^{2} v=\tilde{\lambda}_{A} m_{\tilde{L}, A B}^{2} v_{B}$

To avoid the excessive effort involved in developing the basis independent formalism, we have pursued the analysis by making a fixed choice of the $L_{A}$ field basis, while distinguishing between the free and constrained parameters phenomenologically. Although the choice $v_{i}=0$ is perfectly admissible, this biases the exploration of the parameter space. Upon working in the basis choice $v_{i} \neq 0$, the constraints from the tree and loop level contributions to the neutrino mass matrix can be implemented in several ways. Since the tree contribution is proportional to $\tilde{\lambda}_{i}^{\prime} \equiv \tilde{\lambda}_{i}-\lambda v_{i} / v_{d}$, we choose to regard $\tilde{\lambda}_{i}$ as free parameters and assign by hand $v_{i}$ consistently with the restricted range of variation for $\tilde{\lambda}_{i}^{\prime}$. The basic relation between the gauge boson mass and the electroweak breaking mass scale, $v^{2} \equiv v_{u}^{2}+v_{d}^{2}+v_{i}^{2}=v_{u}^{2}+\hat{v}_{d}^{2}$, is then implemented by using the parameterization of the Higgs-sneutrinos VEVs

$$
v_{d}=\frac{v_{u}}{\tan \beta}=\frac{v}{\left(1+\sigma_{i}^{2}+\tan ^{2} \beta\right)^{\frac{1}{2}}}, \quad v_{i}=\sigma_{i} v_{d}, \quad\left[\sigma_{i}=\frac{\tilde{\lambda}_{i}-\tilde{\lambda}_{i}^{\prime}}{\lambda}\right],
$$

where we retain the usual definition for the ratio of VEVs, $\tan \beta=v_{u} / v_{d}$. At the one-loop level, the contributions to the neutrino mass matrix from the neutralino-slepton exchange Feynman diagram with a double mass insertion, as given by Fig. 1(B) and Eq. (III.54) of our previous work [4], bounds the misalignment parameter $|B \wedge v|$, or equivalently the effective parameters $\eta_{i} \equiv \frac{B_{i}}{B}-\frac{v_{i}}{v_{d}}=\frac{A_{\tilde{\lambda}_{i}} \tilde{\lambda}_{i}}{A_{\lambda} \lambda}-\frac{v_{i}}{v_{d}}$. With the restrictions from the neutrino masses set on $\tilde{\lambda}_{i}^{\prime}$ and $\eta_{i}$, the parameters $v_{i}$ and $A_{\tilde{\lambda}_{i}}$ are explicitly determined in terms of $\tilde{\lambda}_{i}$. It is important to note that once the equations of motion are satisfied, the conditions $\tilde{\lambda}_{A} \propto v_{A}$ and $B_{A} \equiv A_{\tilde{\lambda}_{A}} \tilde{\lambda}_{A} \propto v_{A}$ entail the condition on the squared mass matrix $m_{\tilde{L}, A B}^{2} v_{B} \propto v_{A}$. It then follows that fixing $\tilde{\lambda}_{i}^{\prime}$ and $\eta_{i}$ still leaves the freedom of choosing the slepton mass parameters $m_{\tilde{L}_{i}}^{2}$. Because of the strongly suppressed values $\tilde{\lambda}_{i}^{\prime}<<1$ and $\eta_{i}<<1$ imposed by the neutrino masses, the precise values assigned to $\tilde{\lambda}_{i}^{\prime}$ and $\eta_{i}$ have little effect on the final results.

We shall develop the study of the regular vacuum solution of the scalar sector in terms of the neutral scalar field VEVs $v_{d}, v_{u}, x$ and $v_{i}$. A necessary condition for stability is obtained by testing for the absence of saddle points of the scalar potential along the charged and neutral boson field directions in the field space. This condition is equivalent to the requirement that the squared mass matrices for the charged and neutral scalar bosons are free from negative squared mass tachyonic eigenvalues. To simplify the discussion we shall assign finite values for the lepton number violating parameters (coupling constants and VEVs) one 
at a time for each lepton number flavor, so that finite coupling constants $\tilde{\lambda}_{i}, A_{\tilde{\lambda}_{i}}, m_{H_{d} \tilde{L}_{i}}^{2}$ and finite VEVs, $v_{i}$, are assigned for a fixed flavor index $i$. We thus neglect the quadratic terms of the form $\tilde{\lambda}_{i} \tilde{\lambda}_{j}, v_{i} v_{j}, \tilde{\lambda}_{i} v_{j}, \cdots$, with $i \neq j$. The single dominant coupling constant assumption is not too restrictive as long as the lepton number violating contributions are small relative to those of the lepton number conserving contributions. To assess the validity of this approximation, one could envision using the variant prescription selecting some linear combinations of parameters in the $S U(4)$ group of the $L_{A}$ fields.

The structure of the scalar mass matrices depends on the way in which one implements the equations of motion. Since the scalar potential of the NMSSM is given by a polynomial in the VEVs of the neutral fields, it is impossible, in general, to obtain analytic formulae for the said VEVs. Instead, we follow the practical procedure in which one eliminates the soft mass parameters $m_{H_{u}}^{2}, m_{H_{d}}^{2}, m_{S}^{2}$ via the minimization conditions for the VEVs of electrically neutral fields $v_{u}, v_{d}$, and $x$, respectively. For the sneutrino $\mathrm{VEVs}, v_{i}$, however, we need to specify beforehand our choice for the independent mass parameters, because of the presence of the off-diagonal and diagonal type sleptons mass parameters for the combination $\left(H_{d}, \tilde{L}_{i}\right)$, namely $m_{H_{d} \tilde{L}_{i}}^{2}$ and $m_{\tilde{L}, i j}^{2}$. The freedom in solving the field equations (or equations of motion) for the sneutrinos $\tilde{\nu}_{i}$ could be used to eliminate the mass parameters $m_{H_{d} \tilde{L}_{i}}^{2}$, which would then leave us with $m_{\tilde{L}_{i}}^{2}$ as free parameters. We call this option in the following as our prescription I. Alternatively, we could eliminate the mass parameters $m_{\tilde{L}_{i}}^{2}$, and hence use $m_{H_{d} \tilde{L}_{i}}^{2}$ as free parameters. We call this option in the following as prescription II. These prescriptions only differ in the way one treats the input data for the soft masses. While prescription II is perhaps more natural, since $m_{H_{d} \tilde{L}_{i}}^{2}$ are lepton number violating parameters on the same footing as the soft parameters $A_{\tilde{\lambda}_{i}}$, this has the drawback of introducing inverse powers of the parameters $v_{i}$, which are expected to be small. In any case the results in prescription II are readily obtained from those in prescription I by substituting the expression for $m_{\tilde{L}_{i}}^{2}$ implied by the equations of motion for $\tilde{\nu}_{i}$, which we explicitly provide in Eq. (A.9) of Appendix A. For definiteness, we quote below the formulae obtained with prescription I.

\section{Mass Matrices of Scalar Bosons}

The lepton number violating term proportional to $\tilde{\lambda}_{i}$ in the superpotential (A.2) produces mixing between the charged Higgs bosons and scalar leptons [4]. Similarly, there will 
be mixing between the charginos and the charged leptons. These mixings can be studied through the appropriate mass matrices. Here we discuss the mass matrices of charged Higgs bosons/charged scalar leptons, and neutral Higgs bosons/ neutral scalar leptons, respectively. The mass matrices for charginos/charged leptons and neutralinos/neutral leptons are discussed in the next subsection. The field basis for the charged Higgs-slepton scalar modes is denoted by the column vectors

$$
\Phi_{c h}=\left(H_{d}^{-\star}, H_{u}^{+}, \tilde{e}_{i}^{\star}, \tilde{e}_{i}^{c}\right), \Phi_{c h}^{\dagger}=\left(H_{d}^{-}, H_{u}^{+\star}, \tilde{e}_{i}, \tilde{e}_{i}^{c \star}\right)
$$

with the mass term in the Lagrangian given by

$$
-L_{\text {mass }}=\Phi_{c h}^{\dagger} M_{c h}^{2} \Phi_{c h}+H . c .
$$

After a straightforward calculation, we obtain the mass squared matrix for the charged scalars whose elements can be written as

$$
\begin{aligned}
& M_{H_{d} H_{d}^{\star}}^{2}=\frac{v_{u}}{2 v_{d}}\left(-2 \mu_{d u}^{2}+g_{2}^{2} v_{d} v_{u}-2 \lambda^{2} v_{d} v_{u}+2 A_{\lambda} \lambda x+2 \kappa \lambda x^{2}\right)-\frac{v_{u} x v_{i} \tilde{\lambda}_{i}}{v_{d}^{2}}\left(A_{\tilde{\lambda}_{i}}+\kappa x\right)+\frac{v_{u} v_{i} \mu_{i u}^{2}}{v_{d}^{2}} \\
& +\frac{v_{i}^{2}}{4 v_{d}^{2}}\left(4 m_{\tilde{L}_{i}}^{2}+g_{1}^{2} v_{d}^{2}-g_{2}^{2} v_{d}^{2}-g_{1}^{2} v_{u}^{2}-g_{2}^{2} v_{u}^{2}+4 \tilde{\lambda}_{i}^{2} v_{u}^{2}+4 \tilde{\lambda}_{i}^{2} x^{2}\right) \\
& +\frac{v_{i}^{4}}{4 v_{d}^{2}}\left(g_{1}^{2}+g_{2}^{2}\right)+\lambda_{d j i} \lambda_{d k i} v_{j} v_{k} \\
& M_{H_{d} H_{u}}^{2}=\frac{g_{2}^{2} v_{d} v_{u}}{2}-\lambda^{2} v_{d} v_{u}+\lambda x\left(A_{\lambda}+\kappa x\right)-\lambda \tilde{\lambda}_{i} v_{u} v_{i}-\mu_{d u}^{2} \\
& M_{H_{d} \tilde{e}_{i}^{\star}}^{2}=\lambda \tilde{\lambda}_{i} x^{2}+\frac{g_{2}^{2} v_{d} v_{i}}{2}-\lambda_{d A j} \lambda_{B i j} v_{A} v_{B} \\
& M_{H_{d} \tilde{e}_{i}^{c}}^{2}=-\lambda_{d A i} \tilde{\lambda}_{A} v_{u} x+A_{d A i}^{\lambda} \lambda_{d A i} v_{A} \\
& M_{H_{u}^{\star} H_{u}}^{2}=\frac{v_{d}}{2 v_{u}}\left(-2 \mu_{d u}^{2}+g_{2}^{2} v_{d} v_{u}-2 \lambda^{2} v_{d} v_{u}+2 A_{\lambda} \lambda x+2 \kappa \lambda x^{2}\right) \\
& +\frac{v_{i}}{v_{u}}\left(-\mu_{i u}^{2}-2 \lambda \tilde{\lambda}_{i} v_{d} v_{u}+x \tilde{\lambda}_{i}\left(A_{\tilde{\lambda}_{i}}+\kappa x\right)\right)+\frac{\left(g_{2}^{2}-2 \tilde{\lambda}_{i}^{2}\right) v_{i}^{2}}{2}, \\
& M_{H_{u}^{\star} \tilde{e}_{i}^{\star}}^{2}=-\mu_{i u}^{2}-\lambda \tilde{\lambda}_{i} v_{d} v_{u}+x \tilde{\lambda}_{i}\left(A_{\tilde{\lambda}_{i}}+\kappa x\right)+\frac{v_{u} v_{i}}{2}\left(g_{2}^{2}-2 \tilde{\lambda}_{i}^{2}\right), \\
& M_{H_{u}^{\star} \tilde{e}_{i}^{c}}^{2}=\lambda_{B A i} \tilde{\lambda}_{A} x v_{B}, \\
& M_{\tilde{e}_{i} \tilde{e}_{j}^{\star}}^{2}=m_{\tilde{L}, i j}^{2}+\frac{1}{4}\left[\left(g_{1}^{2}-g_{2}^{2}\right)\left(v_{d}^{2}-v_{u}^{2}\right)+\left(g_{1}^{2}+g_{2}^{2}\right) v_{i}^{2}+4 \tilde{\lambda}_{i}^{2} x^{2}\right] \delta_{i j}+\lambda_{A i k} \lambda_{B j k} v_{A} v_{B}, \\
& M_{\tilde{e}_{i} \tilde{e}_{j}^{c}}^{2}=\tilde{\lambda}_{A} v_{u} \lambda_{A i j} x-A_{A i j}^{\lambda} \lambda_{A i j} v_{A} \\
& M_{\tilde{e}_{i}^{c^{\star \star}} \tilde{e}_{j}^{c}}^{2}=m_{\tilde{E}^{c}, i j}^{2}+\frac{1}{2} g_{1}^{2}\left(-v_{d}^{2}-v_{i}^{2}+v_{u}^{2}\right) \delta_{i j}+\lambda_{A B i} \lambda_{A B^{\prime} j} v_{B} v_{B^{\prime}} .
\end{aligned}
$$

For completeness, we have quoted the results for general bases of the slepton fields. The above formulae agree with Ref. [34] for the MSSM with R parity violation. 
We now consider the neutral spin-0 Higgs-sneutrino fields whose real and imaginary parts carry positive and negative $\mathrm{CP}$ quantum numbers. The field basis for the $\mathrm{CP}$-even and $\mathrm{CP}$ odd Higgs-sneutrino scalar modes are defined by the Lagrangian mass terms as

$$
\begin{aligned}
-L_{\text {mass }} & =\Phi_{\text {neut }}^{\dagger} M_{\text {neut }}^{2} \Phi_{\text {neut }}+H . c . \\
& =\Phi_{s, i}^{\dagger} M_{s, i j}^{2} \Phi_{s, j}+\Phi_{p, i}^{\dagger} M_{p, i j}^{2} \Phi_{p, j}
\end{aligned}
$$

where the neutral spin-0 fields have the decomposition

$$
\Phi_{n e u t, i}=\frac{1}{\sqrt{2}}\left(\Phi_{s, i}+i \Phi_{p, i}\right), \quad \Phi_{n e u t, i}=\left(H_{d}^{0}, H_{u}^{0}, S, \tilde{\nu}_{j}\right) .
$$

The mass squared matrix for CP-even scalars in the prescription I, using the equations of motion for $\tilde{\nu}_{i}$ to eliminate the mass parameter $m_{H_{d} \tilde{L}_{i}}^{2}$ with $m_{\tilde{L}_{i}}^{2}$ as free parameters, is given by

$$
\begin{aligned}
M_{s, d d}^{2}= & \frac{1}{v_{d}}\left[4 G_{+}^{2} v_{d}^{3}+v_{u} x\left(A_{\lambda} \lambda+\kappa \lambda x\right)\right]-\frac{v_{u} x v_{i}}{v_{d}^{2}}\left(A_{\tilde{\lambda}_{i}} \tilde{\lambda}_{i}+\kappa \tilde{\lambda}_{i} x\right) \\
& +\frac{v_{i}^{2}}{v_{d}^{2}}\left(m_{\tilde{L}_{i}}^{2}+2 G_{+}^{2}\left(v_{d}^{2}-v_{u}^{2}\right)+\tilde{\lambda}_{i}^{2}\left(v_{u}^{2}+x^{2}\right)\right)+\frac{2 G_{+}^{2} v_{i}^{4}}{v_{d}^{2}} \\
M_{s, d u}^{2}= & -4 G_{+}^{2} v_{d} v_{u}+2 \lambda^{2} v_{d} v_{u}-A_{\lambda} \lambda x-\kappa \lambda x^{2}+2 \lambda \tilde{\lambda}_{i} v_{u} v_{i} \\
M_{s, d S}^{2}= & -A_{\lambda} \lambda v_{u}+2 \lambda\left(\lambda v_{d}-\kappa v_{u}\right) x+2 \lambda \tilde{\lambda}_{i} x v_{i} \\
M_{s, d \tilde{\nu}_{i}=}^{2}= & \frac{1}{v_{d}}\left[v_{u} x\left(A_{\tilde{\lambda}_{i}} \tilde{\lambda}_{i}+\kappa \tilde{\lambda}_{i} x\right)-\left(m_{\tilde{L}_{i}}^{2}-2 G_{+}^{2}\left(v_{d}^{2}+v_{u}^{2}\right)+\tilde{\lambda}_{i}^{2}\left(v_{u}^{2}+x^{2}\right)\right) v_{i}\right. \\
& \left.-2 G_{+}^{2} v_{i}^{3}\right] \\
M_{s, u u}^{2}= & \frac{1}{v_{u}}\left[4 G_{+}^{2} v_{u}^{3}+v_{d} x\left(A_{\lambda} \lambda+\kappa \lambda x\right)+x\left(A_{\tilde{\lambda}_{i}} \tilde{\lambda}_{i}+\kappa \tilde{\lambda}_{i} x\right) v_{i}\right] \\
M_{s, u S}^{2}= & -A_{\lambda} \lambda v_{d}+2\left(-\left(\kappa \lambda v_{d}\right)+\left(\lambda^{2}+\tilde{\lambda}_{i}^{2}\right) v_{u}\right) x+\left(-A_{\tilde{\lambda}_{i}} \tilde{\lambda}_{i}-2 \kappa \tilde{\lambda}_{i} x\right) v_{i}, \\
M_{s, u \tilde{\nu}_{i}}^{2}= & 2 \lambda \tilde{\lambda}_{i} v_{d} v_{u}-x\left(A_{\tilde{\lambda}_{i}} \tilde{\lambda}_{i}+\kappa \tilde{\lambda}_{i} x\right)+2\left(-2 G_{+}^{2}+\tilde{\lambda}_{i}^{2}\right) v_{u} v_{i} \\
M_{s, S S}^{2}= & \frac{A_{\lambda} \lambda v_{d} v_{u}}{x}+x\left(-A_{\kappa} \kappa+4 \kappa^{2} x\right)+\frac{A_{\tilde{\lambda}_{i}} \tilde{\lambda}_{i} v_{u} v_{i}}{x} \\
M_{s, S \tilde{\nu}_{i}}^{2}= & -A_{\tilde{\lambda}_{i}} \tilde{\lambda}_{i} v_{u}+2 \lambda \tilde{\lambda}_{i} v_{d} x-2 \kappa \tilde{\lambda}_{i} v_{u} x+2 \tilde{\lambda}_{i}^{2} x v_{i} \\
M_{s, \tilde{\nu}_{i} \tilde{\nu}_{i}}^{2}= & m_{\tilde{L}_{i}}^{2}+2 G_{+}^{2}\left(v_{d}^{2}-v_{u}{ }^{2}\right)+\tilde{\lambda}_{i}^{2}\left(v_{u}^{2}+x^{2}\right)+6 G_{+}^{2} v_{i}^{2}
\end{aligned}
$$

and for the CP-odd modes by

$$
\begin{aligned}
M_{p, d d}^{2}= & \frac{v_{u} x\left(A_{\lambda} \lambda+\kappa \lambda x\right)}{v_{d}}-\frac{v_{u} x\left(A_{\tilde{\lambda}_{i}} \tilde{\lambda}_{i}+\kappa \tilde{\lambda}_{i} x\right) v_{i}}{v_{d}^{2}} \\
& +\frac{\left(m_{\tilde{L}_{i}}^{2}+2 G_{+}^{2}\left(v_{d}^{2}-v_{u}^{2}\right)+\tilde{\lambda}_{i}^{2}\left(v_{u}^{2}+x^{2}\right)\right) v_{i}^{2}}{v_{d}{ }^{2}}+\frac{2 G_{+}^{2} v_{i}{ }^{2}}{v_{d}{ }^{2}}
\end{aligned}
$$




$$
\begin{aligned}
M_{p, d u}^{2}= & x\left(A_{\lambda} \lambda+\kappa \lambda x\right), \\
M_{p, d S}^{2}= & v_{u}\left(A_{\lambda} \lambda-2 \kappa \lambda x\right), \\
M_{p, d \tilde{\nu}_{i}}^{2}= & \frac{v_{u} x\left(A_{\tilde{\lambda}_{i}} \tilde{\lambda}_{i}+\kappa \tilde{\lambda}_{i} x\right)}{v_{d}}-\frac{\left(m_{\tilde{L}_{i}}^{2}+2 G_{+}^{2}\left(v_{d}^{2}-v_{u}^{2}\right)+\tilde{\lambda}_{i}^{2}\left(v_{u}^{2}+x^{2}\right)\right) v_{i}}{v_{d}} \\
& -\frac{2 G_{+}^{2} v_{i}^{3}}{v_{d}}, \\
M_{p, u u}^{2}= & \frac{v_{d} x\left(A_{\lambda} \lambda+\kappa \lambda x\right)}{v_{u}}+\frac{x\left(A_{\tilde{\lambda}_{i}} \tilde{\lambda}_{i}+\kappa \tilde{\lambda}_{i} x\right) v_{i}}{v_{u}}, \\
M_{p, u S}^{2}= & v_{d}\left(A_{\lambda} \lambda-2 \kappa \lambda x\right)+\left(A_{\tilde{\lambda}_{i}} \tilde{\lambda}_{i}-2 \kappa \tilde{\lambda}_{i} x\right) v_{i}, \\
M_{p, u \tilde{\nu}_{i}}^{2}= & x\left(A_{\tilde{\lambda}_{i}} \tilde{\lambda}_{i}+\kappa \tilde{\lambda}_{i} x\right), \\
M_{p, S S}^{2}= & \left(4 \kappa \lambda v_{d} v_{u}+\frac{A_{\lambda} \lambda v_{d} v_{u}}{x}+3 A_{\kappa} \kappa x\right)+\frac{v_{u}\left(A_{\tilde{\lambda}_{i}} \tilde{\lambda}_{i}+4 \kappa \tilde{\lambda}_{i} x\right) v_{i}}{x}, \\
M_{p, S \tilde{\nu}_{i}}^{2}= & v_{u}\left(A_{\tilde{\lambda}_{i}} \tilde{\lambda}_{i}-2 \kappa \tilde{\lambda}_{i} x\right), \\
M_{p, \tilde{\nu}_{i} \tilde{\nu}_{i}}^{2}= & m_{\tilde{L}_{i}}^{2}+2 G_{+}^{2}\left(v_{d}^{2}-v_{u}^{2}\right)+\tilde{\lambda}_{i}^{2}\left(v_{u}^{2}+x^{2}\right)+2 G_{+}^{2} v_{i}^{2} .
\end{aligned}
$$

The above formulae agree in the case of vanishing sneutrino VEVs, $v_{i}=0$, with those obtained in our previous work [4].

The change of basis to the would-be Goldstone bosons in charged and neutral sectors is implemented through the transformation

$$
\begin{aligned}
\left(\begin{array}{c}
G^{+} \\
h^{+}
\end{array}\right) & =\mathcal{R}_{\beta}\left(\begin{array}{c}
H_{d}^{-\star} \\
H_{u}^{+}
\end{array}\right),\left(\begin{array}{c}
G^{0} \\
A^{0}
\end{array}\right)=\mathcal{R}_{\beta}\left(\begin{array}{l}
H_{d I} \\
H_{u I}
\end{array}\right), \\
\mathcal{R}_{\beta} & =\left(\begin{array}{cc}
\cos \beta & -\sin \beta \\
\sin \beta & \cos \beta
\end{array}\right) .
\end{aligned}
$$

In order to project out the CP-odd modes and the massless Goldstone mode, $G^{0}$, one simply needs to apply on the 2-dimensional Higgs bosons subspace the $2 \times 2$ matrix rotation $\left(H_{d I}, H_{u I}\right)^{T} \rightarrow\left(G^{0}, A\right)^{T}=\mathcal{R}_{\beta}\left(H_{d I}, H_{u I}\right)^{T}$, where the label $I$ refers to the imaginary part of the fields, namely $H_{d I}=\sqrt{2} \Im\left(H_{d}\right)$. The gauge basis for the physical CP-odd scalar fields is given by $\left(A, \Im(S), \Im\left(\tilde{\nu}_{i}\right)\right)^{T}=\hat{\mathcal{R}}_{\beta}\left(H_{d I}, H_{u I}, S_{I}\right)^{T}$, where $\hat{\mathcal{R}}_{\beta}=\operatorname{diag}\left(\mathcal{R}_{\beta}, 1\right)$. In the basis with $v_{i} \neq 0$, the zero eigenvalue Nambu-Goldstone neutral and electrically charged bosons, defined by the conditions $M_{p, i j}^{2} G_{j}^{0}=0$ and $M_{c h, i j}^{2} G_{j}^{+}=0$, are given in our current choice of basis by the column vectors $G^{0}=\left(v_{d},-v_{u}, 0, v_{i}\right)^{T}$ and $G^{-}=\left(v_{d},-v_{u}, v_{i}, 0\right)^{T}$. The mass eigenstate fields are defined in terms of the mixing matrices which diagonalize the squared mass matrices as

$$
\left(\Phi_{s, p ; I}\right)_{m a s s}=U_{s, p ; I i} \Phi_{s, p ; i},\left[U_{s, p} M_{s, p}^{2} U_{s, p}^{T}=\left(M_{s, p}^{2}\right)_{d i a g}\right]
$$


Among the variety of observables of use in testing the scalar sector (partial decay rates $\left.Z \rightarrow H_{I}+H_{J}, Z \rightarrow H_{I}+A_{J}, Z \rightarrow \tilde{\chi}_{l}^{0}+\tilde{\chi}_{m}^{0}\right)$ we mention, for later reference, the ratio of $Z$ boson vertex couplings $Z Z H_{I}$ to the Higgs bosons defined by $\xi_{Z Z H_{I}}=\left(\frac{g_{Z Z H_{I}}}{g_{Z Z h}^{S M}}\right)^{2}=$ $\left[\left(U_{s}^{T}\right)_{1 I} \cos \beta+\left(U_{s}^{T}\right)_{2 I} \sin \beta\right]^{2}$.

\section{Mass Matrices for Neutralinos and Charginos}

The mass matrix for the coupled system of neutralino and neutrino fields has been calculated in our previous paper [4]. Since the formulae for this matrix are rather complicated and were given in full form there, we do not reproduce these results here. We shall now concentrate on the mass matrix for the charginos/charged leptons. The mass term in the

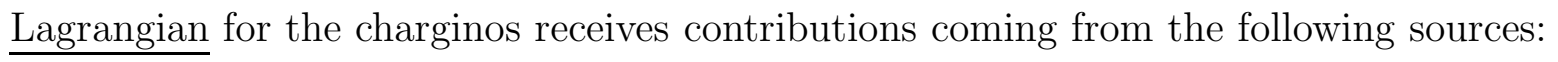

1. Contributions from gauge interactions:

$$
i g \sqrt{2} T_{i j}^{a}\left[\lambda^{a} \psi_{j} \phi_{i}^{*}+H . c\right]
$$

where $T^{a}$ is the generator of the underlying gauge group, $\lambda^{a}$ is the corresponding gaugino, and $\phi_{i}, \psi_{j}$ are the components of the matter superfield.

2. Contributions from the superpotential (in our sign convention):

$$
+\frac{1}{2}\left[\frac{\partial^{2} W}{\partial \phi_{i} \partial \phi_{j}}+H . c .\right],
$$

where $\mathrm{W}$ is the superpotential, and $\phi_{i}$ are the scalar components of a chiral superfield.

3. Soft supersymmetry breaking gaugino masses:

$$
\frac{1}{2} M_{2} \sum_{i} \tilde{\lambda}_{2}^{i} \tilde{\lambda}_{2}^{i}
$$

where "2" here refers to the $S U(2)_{L}$ gauge group, and $i$ are $S U(2)_{L}$ indices. Putting together all these contributions, we can write the mass term for the charginos/charged leptons as

$$
L_{\text {mass }}=-\frac{1}{2}\left(\tilde{\chi}^{+T}, \tilde{\chi}^{-T}\right)\left(\begin{array}{cc}
0 & M_{\tilde{\chi}}^{T} \\
M_{\tilde{\chi}} & 0
\end{array}\right)\left(\begin{array}{c}
\tilde{\chi}^{+} \\
\tilde{\chi}^{-}
\end{array}\right),
$$

where we have chosen the basis

$$
\tilde{\chi}^{+T}=\left(-i \tilde{\lambda}^{+}, \tilde{H}_{u}^{+}, e_{R}^{+}, \mu_{R}^{+}, \tau_{R}^{+}\right),
$$




$$
\begin{aligned}
\tilde{\chi}^{-T} & =\left(-i \tilde{\lambda}^{-}, \tilde{H}_{d}^{-}, e_{L}^{-}, \mu_{L}^{-}, \tau_{L}^{-}\right), \\
\tilde{\lambda}^{ \pm} & =\frac{1}{\sqrt{2}}\left(\tilde{\lambda}_{2}^{1} \mp i \tilde{\lambda}_{2}^{2}\right),
\end{aligned}
$$

with the mass matrix for the charginos/charged leptons given by

$$
M_{\tilde{\chi}}^{2}=\left(\begin{array}{ccccc}
M_{2} & g_{2} v_{u} & 0 & 0 & 0 \\
g_{2} v_{d} & \lambda x & \left(\lambda^{e}\right)_{11} v_{1} & \left(\lambda^{e}\right)_{22} v_{2} & \left(\lambda^{e}\right)_{33} v_{3} \\
g_{2} v_{1} & \tilde{\lambda}_{1} x & -\left(\lambda^{e}\right)_{11} v_{d} & 0 & 0 \\
g_{2} v_{2} & \tilde{\lambda}_{2} x & 0 & -\left(\lambda^{e}\right)_{22} v_{d} & 0 \\
g_{2} v_{3} & \tilde{\lambda}_{3} x & 0 & 0 & -\left(\lambda^{e}\right)_{33} v_{d}
\end{array}\right) .
$$

Here we have assumed that the lepton Yukawa coupling and mass matrices, $\lambda_{j k}^{e}=-M_{j k}^{e} / v_{d}$, are diagonal.

\section{IMPLICATIONS ON SCALAR SECTOR AND VACUUM STABILITY}

Before discussing the scalar sector, we briefly discuss some numerical predictions for the light neutrino masses. The tree level contribution and the supposedly dominant oneloop contribution arising from neutralino-slepton exchange, give a neutrino mass matrix of approximate structure in the lepton generation space, $m_{\nu, i j} \simeq a \tilde{\lambda}_{i}^{\prime} \tilde{\lambda}_{j}^{\prime}+b \eta_{i} \eta_{j}$, $\left[\tilde{\lambda}_{i}^{\prime}=\tilde{\lambda}_{i}-\right.$ $\left.\lambda v_{i} / v_{d}, \eta_{i}=B_{i}^{\prime} / B=B_{i} / B-v_{i} / v_{d}\right]$ where the coefficients $a, b$ are expected to have a smooth dependence on the parameters $\lambda, \tan \beta$, and $x$. The pair of non-vanishing mass eigenvalues of this matrix are expressed in terms of the invariant parameters by

$$
\begin{aligned}
& m_{\nu_{2}}=\frac{b}{2 \tilde{\lambda}^{\prime 2}}\left|\overrightarrow{\tilde{\lambda}^{\prime}} \wedge \vec{\eta}\right|^{2}, m_{\nu_{3}}=a \tilde{\lambda}^{\prime 2}+\frac{b}{\tilde{\lambda}^{\prime 2}}\left(\overrightarrow{\tilde{\lambda}^{\prime}} \cdot \vec{\eta}\right)^{2} \\
& {\left[\tilde{\lambda}^{\prime 2}=\sum_{i} \tilde{\lambda}_{i}^{\prime 2}, \eta^{2}=\sum_{i} \eta_{i}^{2},\left|\overrightarrow{\tilde{\lambda}}^{\prime} \wedge \vec{\eta}\right|^{2}=\frac{1}{2} \sum_{i, j}\left(\tilde{\lambda}_{i}^{\prime} \eta_{j}-\tilde{\lambda}_{j}^{\prime} \eta_{i}\right)^{2}, \overrightarrow{\tilde{\lambda}^{\prime}} \cdot \vec{\eta}=\sum_{i} \tilde{\lambda}_{i}^{\prime} \eta_{i}\right] .}
\end{aligned}
$$

For clarity, we quote the explicit expression for the single non-vanishing neutrino Majorana mass eigenvalue present at the tree level [4]

$$
\begin{aligned}
& m_{\nu_{3}}=\frac{\sigma x^{2} V_{u}^{2}\left(M_{2} / M_{1}+g_{2}^{2} / g_{1}^{2}\right)\left(\tilde{\lambda}_{i}-v_{i} \lambda / v_{d}\right)^{2}}{\tan ^{2} \beta\left[V_{u}^{2}\left(M_{2} / M_{1}+g_{2}^{2} / g_{1}^{2}\right)\left(4 \kappa \lambda x^{2} / \tan \beta+\left(\rho / \tan \beta-\lambda v_{u}\right)^{2}\right)-M_{2} \lambda^{2} x^{2} \sigma\right]}, \\
& {\left[\sigma=2\left(\kappa x-\frac{\rho v_{u}}{x}\right), \rho=-\sum_{A} \tilde{\lambda}_{A} v_{A}, \mu=-\lambda x, V_{u}=\frac{g_{1} v_{u}}{\sqrt{2}}\right] .}
\end{aligned}
$$

We have attempted to extract the values of the coefficients $a$ and $b$ from numerical calculations of the tree level formula, Eq. (III.2), and of the one-loop amplitude in Eq. (III.54) 
of our previous work [4]. Using the reference set of parameters given in the caption of Fig. 1 with $\tan \beta=2$, gives us the following order of magnitude estimates: $a \approx 10 \mathrm{GeV}, b \approx 10^{-1}$ $\mathrm{GeV}$. For these predictions to be compatible with the limit on the neutrino mass, $\left|m_{\nu}\right|<1$ $\mathrm{eV}$, we need to impose $\lambda_{i}^{\prime}<10^{-5}, \eta_{i}<10^{-4}$. We have not attempted a global fit to the neutrino experimental data but note that this could be done by following a similar treatment as that used for the MSSM with bilinear R parity violation [35].

\section{A. Numerical Study of Scalar Sector}

In order to have a reliable semi-quantitative description of the NMSSM, we have added to the tree level scalar potential the lepton number conserving one-loop contributions from the top quark and stop pair of squarks. The corrections to the neutral scalar mass matrix are obtained by evaluating Eq. (II.25) using a standard procedure [32], so we refrain from quoting the explicit formulas. The results of our numerical calculations for the mass spectra of the scalar and sfermion modes are shown in Figs. 1, 2 and 3 as single parameter plots displaying the dependence on the lepton number violating couplings $\tilde{\lambda}_{i}$. These results were obtained by considering the reference set of natural input parameters used in [32], which are detailed in the caption of Fig. 1. Although our analytical formulae include the trilinear lepton number violating interactions with coupling constants $\lambda_{i j k}, \lambda_{i j k}^{\prime}$, in our numerical calculations we have retained only the lepton number violating couplings $\tilde{\lambda}_{i}$.

In Fig. 1 we present the results of our numerical calculations for the masses of the lightest neutral and charged scalar modes, and neutralino and chargino modes as a function of the lepton number violating coupling, which we take to vary within the range $\tilde{\lambda}_{i} / \lambda \in[0,1]$. At small $\tan \beta=O(1)$, the modifications induced by $\tilde{\lambda}_{i}$ are essentially quantitative. As the coupling increases, the lightest CP-even scalar mass is enhanced and the CP-odd and charged scalar masses are reduced. This effect of enhancement and reduction of masses are clearly seen at large values of $r \equiv x / v$. These results are also sensitive to the value of the $\mathrm{VEV}$ ratio $\tan \beta=v_{u} / v_{d}$. While the constraints on the scalar masses restrict $\tan \beta$ to a

small natural range in the NMSSM, this feature is even more pronounced for large values of the lepton number violating interactions $\tilde{\lambda}_{i} \approx \lambda$. Already for the value $\tan \beta=8$, the mass spectra undergo a qualitative change with large negative contributions to both the neutral and charged scalar bosons causing the vacuum instability signalled by the occurrence of 
tachyons at $\tilde{\lambda}_{i} / \lambda \geq 0.5$. This is explained by inspection of Eq. (II.28) which shows how increasing $v_{i} / v_{d}$ proportionately to $\tilde{\lambda}_{i} / \lambda$ has the same effect as an increase of $\tan \beta$. The allowed intervals for $\tan \beta$ further shrink with increasing $x$. We also present in Fig. 1 the masses of the lowest lying neutralino and chargino states. The results are characterized by a slow growth of the masses with increasing $\tilde{\lambda}_{i} / \lambda$.

The coupling of $Z$ to the Higgs, $\xi_{Z Z H_{1}}$, is found to vary in the intervals $(0.84 \rightarrow$ $0.63),(0.99 \rightarrow 0.91),(0.96 \rightarrow 0.81),(0.99 \rightarrow 0.94)$ as $\tilde{\lambda}_{i} / \lambda$ increases in the the range $(0.1 \rightarrow 1.0)$ for the mass spectra displayed in panels $(a),(b),(c),(d)$ of Fig. 1. For the sake of a qualitative comparison with experiment, we note that the LEP data indicates a correlation between the lightest Higgs boson vector coupling and mass such that $\xi_{Z Z H_{1}}<(0.02-1.0)$ for $M_{H_{1}}=(50-120) \mathrm{GeV}$.

In order to understand the dependence of the scalar mass spectrum on the supersymmetry breaking interactions, we display in Fig. 2 the results obtained for the case $A_{\tilde{\lambda}_{i}}=0$. This choice is made only for illustrative purposes since it violates the alignment condition for the parameters $\eta_{i}$. As compared to our initial choice, $A_{\tilde{\lambda}_{i}} \neq 0$, in Fig. 1 , we see that switching off the supersymmetry breaking trilinear lepton number violating interactions results in an enhanced effect at small $\tan \beta \simeq 2$ but a reduced effect at $\tan \beta \simeq 8$. Indeed, the vacuum instability at $\tan \beta=8$ sets in at larger values of $\tilde{\lambda}_{i} / \lambda$.

In Fig. 3 we display one-parameter plots for the masses of the lowest lying neutral CPeven and CP-odd Higgs bosons as a function of $\tan \beta$ (fixed $x$ ) and as a function of $x$ (fixed $\tan \beta$ ) for the discrete set of values for $\tilde{\lambda}_{i} / \lambda=0.1,0.5,1$. From these results we see that the maximal values of $\tan \beta$ allowed by vacuum stability lie inside the range $5-10$, and that this interval gets further reduced at larger values of the VEV of the singlet field $x$. As a related observation, we note that the allowed interval for $r \equiv x / v \in[1,6]$ shrinks to $r \in[1,2]$ at $\tan \beta=4$ for large enough values of $\tilde{\lambda}_{i}$. Furthermore, we observe that for the fixed values of $r=2.03,4.06$ in the panels $(a),(b)$, corresponding to $x \sqrt{2}=500 \mathrm{GeV}$ and $1 \mathrm{TeV}$, respectively, our results at small $\tilde{\lambda}_{i} / \lambda \simeq 0.1$ join smoothly with those at $\tilde{\lambda}_{i}=0$, as can be seen in Fig. 1 (panels $(a)$ and $(c)$ ) of Ref. [32]. 
(a) $(\mathrm{r}=2.03, \tan \beta=2)$

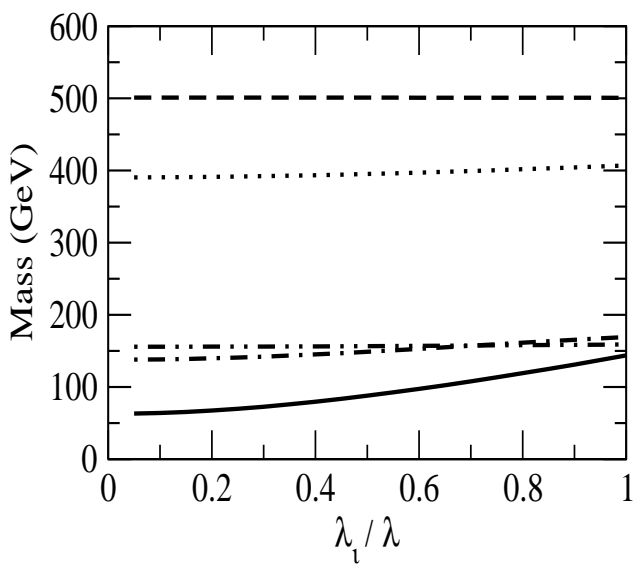

(c) $(\mathrm{r}=2.03, \tan \beta=8)$

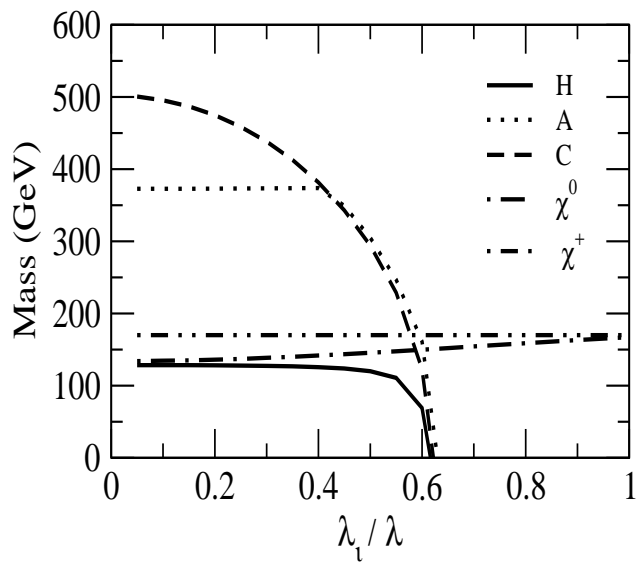

(b) $(r=4.06, \tan \beta=2)$

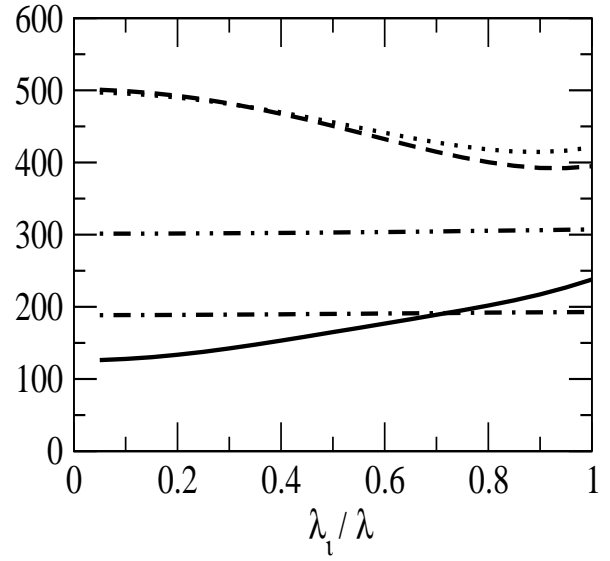

(d) $(r=4.06, \tan \beta=8)$

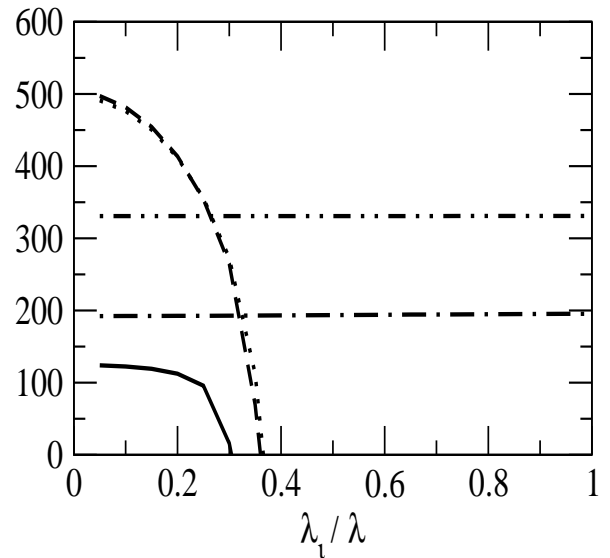

FIG. 1: The masses of lowest lying CP-even and CP-odd neutral scalars, the charged scalars, and the neutralino and chargino states are plotted as a function of $\tilde{\lambda}_{i} / \lambda$. We assume the single lepton number violating dominance hypothesis with a fixed generation $\tilde{\lambda}_{i} \neq 0$ considered one at a time. The curves for $H_{I=1}, A_{I=1}, C_{I=1}, \tilde{\chi}_{l=1}^{0}$ and $\tilde{\chi}_{l=1}^{+}$are drawn in full, dotted, dashed, dot-dash and double-dot dashed lines, respectively, as illustrated in the legend. The upper panels $(a)$ and $(b)$ correspond to $\tan \beta=2$ whereas the lower panels $(c)$ and $(d)$ to $\tan \beta=8$. The left hand panels $(a)$ and $(c)$, and the right hand panels $(b)$ and $(d)$ refer to the parameter $r \equiv x / v=2.03$ and 4.03, respectively. Our choice of the parameters, as given in Ref. [32] (Fig. 1), is described by the values: $\lambda=0.5, \kappa=+0.5, A_{\lambda}=500 \mathrm{GeV}, A_{\kappa}=+250 \mathrm{GeV}$, and $A_{t}^{u}=1 \mathrm{TeV}, m_{\tilde{Q}}=m_{\tilde{U}^{c}}=$ $1 \mathrm{TeV}, Q=300 \mathrm{GeV}$, which enter the tree and one-loop contributions to the scalar potential. In the prescription I in which we work, we set the sneutrino mass parameter at $m_{\tilde{L}_{i}}^{2}=500 \mathrm{GeV}$. The neutralino-neutrino and chargino-lepton mass matrices are evaluated by assuming the relation between the gaugino mass parameters $M_{1}=\frac{k_{1} g_{1}^{2}}{k_{2} g_{2}^{2}} M_{2}$, with $k_{1}=\frac{5}{3}, k_{2}=1$, while using the numerical values $M_{2}=400 \mathrm{GeV}$ and $M_{1}=199.2 \mathrm{GeV}$. The values of parameters $v_{i}$ and $A_{\tilde{\lambda}_{i}}$ are set through the alignment conditions $\tilde{\lambda}_{i}^{\prime} \approx 0, \eta_{i} \approx 0$, whełe $\tilde{\lambda}_{i}^{\prime} \equiv \tilde{\lambda}_{i}-\frac{\lambda v_{i}}{v_{d}}, \eta_{i} \equiv \frac{A_{\tilde{\lambda}_{i}} \tilde{\lambda}_{i}}{A_{\lambda} \lambda}-\frac{v_{i}}{v_{d}} \equiv \frac{B_{i}^{\prime}}{B}, \quad\left[B_{i}^{\prime}=\right.$ $\left.B_{i}-B \frac{v_{i}}{v_{v}}, B_{i}=A_{\tilde{\lambda}_{i}} \tilde{\lambda}_{i}, B=A_{\lambda} \lambda\right]$. 


$$
A_{\lambda_{1}}=0
$$

(a) $(r=2.03, \tan \beta=2)$

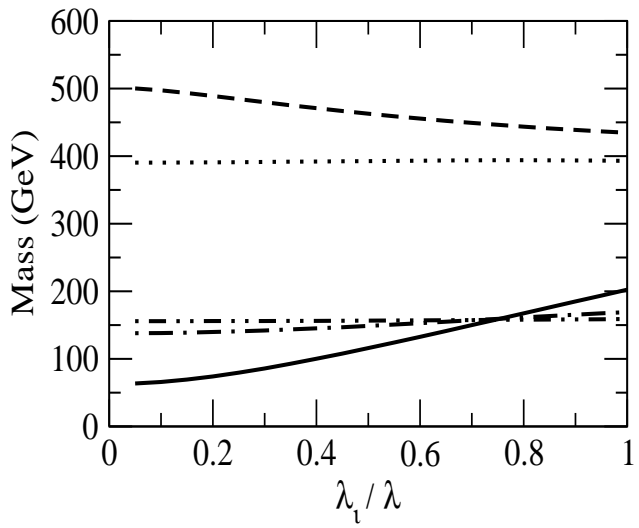

(c) $(\mathrm{r}=2.03, \tan \beta=8)$

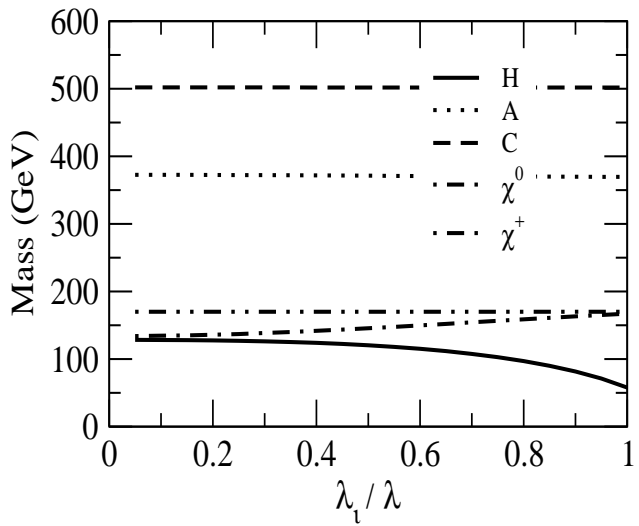

(b) $(r=4.06, \tan \beta=2)$

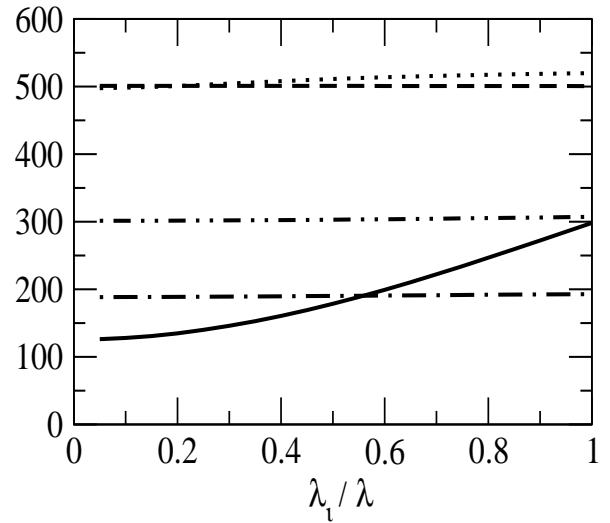

(d) $(r=4.06, \tan \beta=8)$

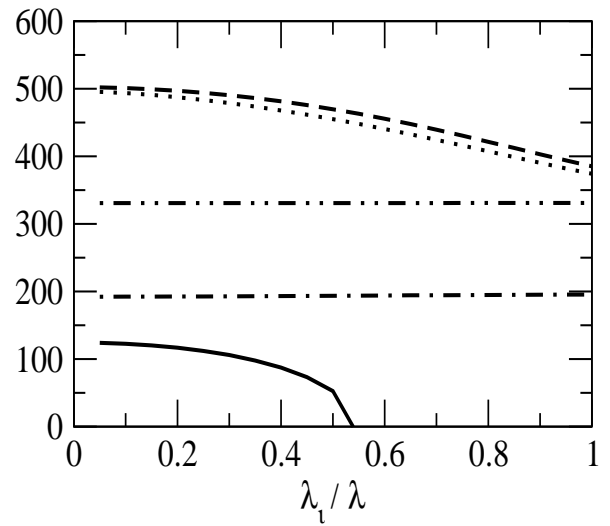

FIG. 2: The masses of the lightest CP-even and CP-odd neutral scalars are plotted as a function of $\tilde{\lambda}_{i} / \lambda$, for fixed lepton generation, under same conditions as in Fig. 1. The single lepton number violating dominance hypothesis is assumed such that finite coupling constants $\tilde{\lambda}_{i}$ of fixed generation are considered one at a time. We use the same set of NMSSM parameters as in Fig. 1, the only change being the vanishing trilinear supersymmetry breaking coupling, $A_{\tilde{\lambda}_{i}}=0$. We have also displayed the lightest neutralino and chargino masses although these are independent of the supersymmetry breaking parameter $A_{\tilde{\lambda}_{i}}$, and are given by the same curves as in Fig. 1. 
(a) $r=2.03$

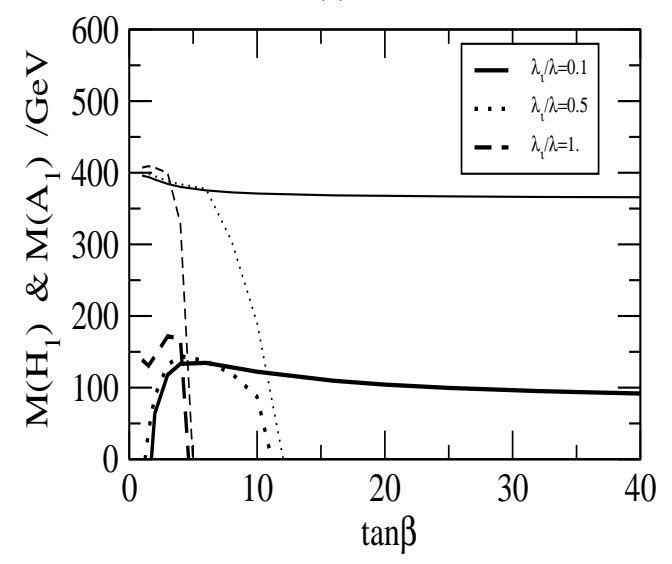

(c) $\tan \beta=2$

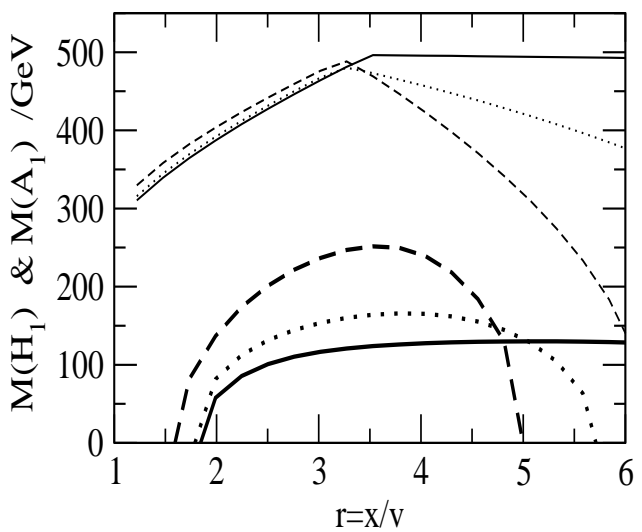

(b) $r=4.06$

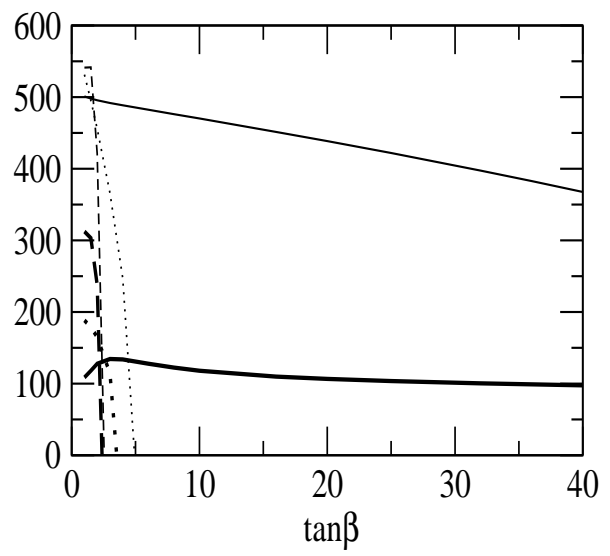

(d) $\tan \beta=4$

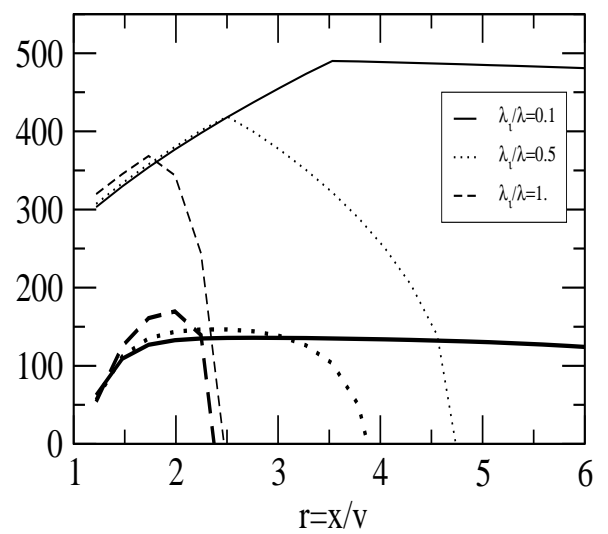

FIG. 3: The masses of the lightest CP-even and CP-odd neutral scalars plotted as a function of $\tan \beta$ at two fixed values of $r=x / v=2.03$ and 4.06 (upper panels $(a)$ and $(b)$ ), corresponding to $x \sqrt{2}=500 \mathrm{GeV}$ and $1 \mathrm{TeV}$, respectively, and as a function of $r=x / v$ at the two fixed values of $\tan \beta=2$ and 4 (lower panels $(c)$ and $(d)$ ). We use the same set of parameters as in Fig. 1 and assume the single lepton number violating dominance hypothesis with a finite $\tilde{\lambda}_{i}$ of fixed generation. The three curves for the modes $H_{I=1}$ (bold) and $A_{I=1}$ (light) are drawn in full, dotted and dashed lines corresponding to the values $\tilde{\lambda}_{i} / \lambda=0.1,0.5,1$. 


\section{CONSTRAINTS FROM UNBOUNDED FROM BELOW DIRECTIONS AND CHARGE AND COLOR BREAKING MINIMA}

\section{A. General Considerations}

The stability of the regular vacuum with respect to the UFB directions and the distant CCB minima in the scalar field space are expected to give useful constraints on the NMSSM parameters. We briefly recall here the main features of the renormalization group approach to this problem which was extensively discussed in the context of MSSM [22]. This approach is particularly useful in the context of a gravity mediation of supersymmetry breaking in grand unified theories (supergravity GUT), where the unknown independent parameters consist of a small set of parameters and one can develop semi-analytic methods. The idea is to select suitable directions in the scalar field space along which the contributions to the scalar potential are dominated by the supersymmetry breaking terms while those from $F$ - and $D$ - terms are smaller and smoothly varying. In practice, the field directions are parameterized in terms of some single real variable, $w$, along which the scalar potential is given by a polynomial in $w$ which receives, for a suitable choice of the parameter phases, negative contributions of $O\left(w^{2}\right)$ or $O\left(w^{3}\right)$ from the supersymmetry breaking trilinear scalar couplings and mass terms, and positive contributions of $O\left(w^{4}\right)$ or higher order from the supersymmetry terms. The higher order positive terms lift the potential upwards at large $w$. Even when absent at the tree level, higher order contributions to the potential, which lift it, always arise at the one-loop order. Requiring the scalar potential along these directions to stay above the regular minimum yields algebraic conditions on the coefficients of the polynomial. Considering, for illustration, the case of the field direction yielding the quartic order potential, $V(w)=A w^{4}+B w^{2}+C$, where the coefficients $A, B, C$ are known algebraic functions of the various parameters, we see that this is an UFB direction if $A=0$. At $A>0$, this direction develops a minimum if $B<0$, as seen by evaluating the extremum with respect to $w^{2}$

$$
w_{\text {min }}^{2}=-\frac{B}{2 A} \Longrightarrow V\left(w_{\text {min }}\right)=-\frac{B^{2}}{4 A}+C .
$$

The regular electroweak symmetry breaking vacuum is stable against decay to the new vacuum solution as long as $V\left(w_{\min }\right)>V_{M I N}$, where $V_{M I N}$ denotes the value at the regular minimum. An improved quantum stability condition can be deduced without a detailed 
knowledge of the one-loop radiative corrections to the potential upon invoking the logarithmic dependence on the masses of states scaled by the running momentum scale $Q$. One needs only to assume that the above inequality involving the tree level scalar potential still holds but with the parameters replaced by renormalization group improved, momentum scale $Q$ dependent, running parameters,

$$
V_{U F B}(w ; Q=\hat{Q})>V_{M I N}\left(w_{0} ; Q=M_{S}\right),
$$

where the arguments $w$ and $Q$ along the field direction are set in the grand unification framework as $w \in\left[m_{W}, M_{X}\right]$ and $\hat{Q}=\operatorname{Max}\left(g_{2} w, \lambda_{t} w, M_{S}\right)$, and those for the regular minimum are set as $w_{0}=\left[v_{u}, v_{d}\right]$ and $Q=M_{S}$, where $v_{u}$ and $v_{d}$ denote the the usual Higgs VEVs, and $M_{S}$ the effective supersymmetry breaking mass scale. Extensive analyses of the vacuum stability constraints have been developed for the MSSM [22]. For the MSSM with broken R-parity, Abel and Savoy [23] inferred lower bounds on the trilinear R-parity violating couplings $\lambda_{i j k}, \lambda_{i j k}^{\prime}$, and Hirsch et al., [24] discussed the implications on the bilinear R-parity violating interactions.

Although a similar programme for the NMSSM with lepton number violation appears to be well motivated, its implementation is substantially complicated by the need to consider two-dimensional field directions which include the singlet field $S=x$ as an independent variable, since there are no obvious correlations between the contributions from the electroweak singlet and non-singlet fields. Another complication stems from the fact that the renormalization group formalism is more complicated. This can be seen from the absence of studies of the dangerous field directions for the NMSSM beyond the preliminary works [36-38]. The progress achieved through the quasi fixed point solution for the running parameters [39, 40] or the numerical studies of physical constraints $[41,42,44]$ are not of direct help to us in the present work. Since our main focus is on the lepton number violation, rather than solving the full-fledged problem, we shall follow a simple phenomenological approach, which we now describe. We assign natural values for the lepton number conserving Yukawa couplings and soft parameters which respect the regular minimum stability, and then examine the effect of increasing the lepton number violating parameters $\tilde{\lambda}_{i}, B_{i}, v_{i}$, consistently with the constraints from the neutrino mass matrix. This approach is similar in spirit to that followed in [24].

The field directions involve suitable subsets of the electrically neutral and charged scalar 
states and the squark states. We continue using the single lepton flavor dominance hypothesis in which the nonvanishing parameters, $\tilde{\lambda}_{i}, A_{\tilde{\lambda}_{i}}, v_{i}$ are finite only for fixed generation labels $i, j, \cdots$. Useful intermediate formulas for the scalar potential of the electrically neutral fields and for the classical equations of motion of the electrically neutral and charged fields are given in Eq. (A.8), Eq. (A.9) and Eq. (A.11) of the Appendix A.

To start with, we need the potential at the regular minimum. With our prescription of eliminating the dependence on the soft mass parameters $m_{H_{u}}^{2}, m_{H_{d}}^{2}, m_{S}^{2}$, and $m_{H_{d} \tilde{L}_{i}}^{2}$ by using the minimization equations with respect to $v_{u}, v_{d}, x$, and $v_{i}$, the value of the scalar potential at the minimum is given by

$$
\begin{aligned}
V_{M I N}\left(v_{u}, v_{d}, x, v_{i}\right) & =-G_{+}^{2}\left(v_{d}^{2}-v_{u}{ }^{2}\right)^{2}-\frac{1}{3}\left[-6 \kappa \lambda v_{d} v_{u} x^{2}+3 \lambda^{2}\left(v_{u}{ }^{2} x^{2}+v_{d}{ }^{2}\left(v_{u}{ }^{2}+x^{2}\right)\right)\right. \\
& \left.+x\left(-3 A_{\lambda} \lambda v_{d} v_{u}+x\left(3 \tilde{\lambda}_{i}^{2} v_{u}{ }^{2}-A_{\kappa} x+3 \kappa^{2} x^{2}\right)\right)\right] \\
& +\left[v_{u} x\left(A_{\tilde{\lambda}_{i}} \tilde{\lambda}_{i}+2 \kappa \tilde{\lambda}_{i} x\right)-2 \lambda \tilde{\lambda}_{i} v_{d}\left(v_{u}{ }^{2}+x^{2}\right)\right] v_{i} \\
& +\left[2 G_{+}^{2}\left(-v_{d}{ }^{2}+v_{u}{ }^{2}\right)-\tilde{\lambda}_{i}^{2}\left(v_{u}{ }^{2}+x^{2}\right)\right] v_{i}{ }^{2}-G_{+}^{2} v_{i}{ }^{4}
\end{aligned}
$$

The above formula is seen to be independent of the slepton-Higgs boson soft mass parame-

ters, so that it holds in the same form in the prescription where one eliminates $m_{\tilde{L}_{i}}^{2}$ rather than $m_{H_{d} \tilde{L}_{i}}^{2}$. We also note that the quartic and quadratic dependence on the VEVs coming from the gauge interactions combine into a term of same form as the minimum value of the scalar potential in the MSSM with bilinear $R$-parity violation

$$
\left(V_{M I N}\right)_{M S S M}=-G_{+}^{2}\left(v_{i}^{2}+v_{d}^{2}-v_{u}^{2}\right)^{2} .
$$

\section{B. Unbounded From Below Directions}

The Higgs-slepton field directions of interest are those which minimize the positive contributions from $F$ - and $D$-terms and maximize the negative contributions from soft masses and trilinear couplings. There are three main unbounded from below directions defined by

$$
\begin{aligned}
& U F B-1: H_{d}=H_{u} \neq 0, S \neq 0 \\
& U F B-2: H_{d} \neq 0, H_{u} \neq 0, \tilde{\nu}_{i} \neq 0, S \neq 0 \\
& U F B-3: \quad H_{d}=0, H_{u} \neq 0, \quad \tilde{\nu}_{i} \neq 0, \tilde{e}_{j}=\tilde{e}_{j}^{c} \neq 0, S \neq 0,[i \neq j] .
\end{aligned}
$$


The singlet field dependence of the potential makes an analytic study intractable. For instance, minimizing the potential with respect to $S$ introduces a non-polynomial dependence with respect to the variables describing the electroweak non-singlet field directions.

We now discuss the three UFB directions in detail. Along the $U F B-1$ direction described by the two variables $\left|H_{d}\right|=\left|H_{u}\right|=w$ and $S=x$, the potential is given by

$$
\begin{aligned}
V_{U F B-1}(w, x) & =A w^{4}+B(x) w^{2}+C(x), \\
A & =\lambda^{2}, B(x)=m_{H_{u}}^{2}+m_{H_{d}}^{2}-2 A_{\lambda} \lambda x+2 \mu_{d u}^{2}+x^{2}\left(2 \lambda(\lambda-\kappa)+\tilde{\lambda}_{i}^{2}\right), \\
C(x) & =\kappa^{2} x^{4}-\frac{2}{3} A_{\kappa} \kappa x^{3}+m_{S}^{2} x^{2} .
\end{aligned}
$$

We have included the dependence on $\mu_{d u}^{2}$ although this parameter is expected to be absent, as already discussed, in the minimal version of the model. For $\lambda \neq 0$, the would-be UFB direction is lifted at large $w$, and features a minimum at $w_{\min }$ unless $B(x)>0$. It is useful to note that $B(x)$ is a quadratic form in $x$ with an extremum at $x_{\min }$ defined by $\partial B /\left.\partial x\right|_{x=x_{\min }}=0 \Longrightarrow x_{\min }=A_{\lambda} \lambda /\left[2 \lambda(\lambda-\kappa)+\tilde{\lambda}_{i}^{2}\right]$. Since this is a minimum provided that $2 \lambda(\lambda-\kappa)+\tilde{\lambda}_{i}^{2}>0$, one can express the condition for the absence of the UFB- 1 direction by the appoximate bound on the soft parameters

$$
B(x) \geq B\left(x_{\text {min }}\right)=m_{H_{u}}^{2}+m_{H_{d}}^{2}-\frac{\left(A_{\lambda} \lambda\right)^{2}}{2 \lambda(\lambda-\kappa)+\tilde{\lambda}_{i}^{2}}+2 \mu_{d u}^{2}>0 .
$$

It is interesting to compare with the corresponding bound in the MSSM, $B\left(x_{\min }\right) \rightarrow m_{H_{u}}^{2}+$ $m_{H_{d}}^{2}+2\left(\mu^{2}-\left(A_{\mu} \mu\right)^{2}\right)$. To obtain the improved constraint, one should determine the position of the potential minimum, $x_{\min }$ and $w_{\min }$, and require the condition $V_{U F B-1}\left(x_{\min }, w_{\min } ; \hat{Q}\right) \geq$ $V_{M I N}\left(x=x_{0}, v_{u}=v \sin \beta, v_{d}=v \cos \beta ; Q=M_{S}\right)$.

The UFB-2 field direction can be conveniently described by the parameterization $v_{u}=$ $w, v_{d}=w \cos \theta, v_{i}=w \sin \theta, x,[\theta \in[0,2 \pi]]$, which is designed to cancel the $D$-terms. The scalar potential is given by the quartic order polynomial in $w$

$$
V_{U F B-2}(w, x, \theta) \equiv A(x, \theta) w^{4}+B(x, \theta) w^{2}+C(x, \theta),
$$

where

$$
\begin{aligned}
A(x, \theta) & =\left(\lambda \cos (\theta)+\tilde{\lambda}_{i} \sin (\theta)\right)^{2}, \\
B(x, \theta) & =\lambda^{2} x^{2}+\tilde{\lambda}_{i}^{2} x^{2}+m_{H_{u}}^{2}-2 x\left(A_{\lambda} \lambda+\kappa \lambda x\right) \cos (\theta) \\
& +\left(\lambda^{2} x^{2}+m_{H_{d}}^{2}\right) \cos ^{2}(\theta)-2 x\left(A_{\tilde{\lambda}_{i}} \tilde{\lambda}_{i}+\kappa \tilde{\lambda}_{i} x\right) \sin (\theta)
\end{aligned}
$$




$$
\begin{aligned}
& +\left(\tilde{\lambda}_{i}^{2} x^{2}+m_{\tilde{L}_{i}}^{2}\right) \sin ^{2}(\theta)+\lambda \tilde{\lambda}_{i} x^{2} \sin (2 \theta), \\
C(x, \theta) & =\frac{-2 A_{\kappa} \kappa x^{3}}{3}+\kappa^{2} x^{4}+x^{2} m_{S}^{2} .
\end{aligned}
$$

Since the UFB-2 direction coincides with the UFB- 1 direction at $\theta=0$, to determine whether one avoids a distant minimum along $w$ it is only necessary to test the condition that the coefficient $B(x, \theta)<0$ at some finite $\theta$. Inspection of Eq. (IV.10) for $B(x, \theta)$ shows that the condition $B(x, \theta=0)>0$ is sensitive to the signs of parameters $m_{H_{d}}^{2}, m_{H_{u}}^{2}$ and $A_{\lambda}$, while the condition $B(x, \theta)>0$ at finite $\theta$ is sensitive to the signs of $A_{\tilde{\lambda}_{i}}$ and $m_{\tilde{L}_{i}}^{2}$. To achieve $B(x, 0)>0$, it is more favorable to restrict to the choice $A_{\lambda}<0$. We now attempt to assign by hand typical values to the relevant free coupling and mass parameters, and determine numerically whether the lepton number violating interactions can drive $B(x, \theta)$ to negative values if it started from a positive value at $\theta=0$. The dependence on $\theta$ is displayed in Fig. 4 for typical values of the input parameters which are specified in the caption of that figure. We see from these results that for trilinear couplings $A_{\tilde{\lambda}_{i}}$ of same negative sign as $A_{\lambda}$, the $\tilde{\lambda}_{i}$ interactions give significant positive sign contributions to $B(x, \theta)$ which avoid the occurrence of dangerous minima along $w$ at finite $\theta$. By contrast, choosing triscalar couplings of opposite sign, $A_{\tilde{\lambda}_{i}}>0$, always drives $B(x, \theta)$ to negative values at finite $\theta$. We note that a dependence on $\theta$ of similar type is found with various other choices of $x$ and that changing the sign of $\tilde{\lambda}_{i}$ leaves the potential unchanged up to the replacement, $\theta \rightarrow \pi-\theta$. We, thus, conclude that the lepton number violating couplings, $\tilde{\lambda}_{i}$ and $A_{\tilde{\lambda}_{i}}$, have the ability to remove or induce the dangerous minima at finite $\theta$ depending on whether $A_{\tilde{\lambda}_{i}}$ is negative or positive, namely, of same or opposite sign to $A_{\lambda}$. We note that the case with $A_{\lambda}$ and $A_{\tilde{\lambda}_{i}}$ of opposite signs clashes with the universality of supersymmetry breaking and is unlikeky to occur in the context of grand unified theories.

The UFB-3 direction can be conveniently described by the parameterization $v_{u}=$ $\sigma w^{2}, e_{j}=e_{j}^{c}=\sigma w, v_{i}=\sigma w\left(1+w^{2}\right)^{\frac{1}{2}}[i \neq j]$, designed to cancel the $D$-terms, while using $\sigma=\frac{\lambda x}{\lambda_{j}^{e}}$, in order to satisfy the $F$-term flatness condition $W_{H_{d}}=0$. The potential along this direction is given by

$$
V_{U F B-3}(w, x)=-\frac{2}{3} A_{\kappa} \kappa x^{3}+\kappa^{2} x^{4}+\tilde{\lambda}_{i}^{2} w^{6} \sigma^{4}+\tilde{\lambda}_{i}^{2} w^{8} \sigma^{4}+w^{3}\left(-2 A_{\tilde{\lambda}_{i}} \tilde{\lambda}_{i} \sqrt{1+w^{2}} x \sigma^{2}\right.
$$




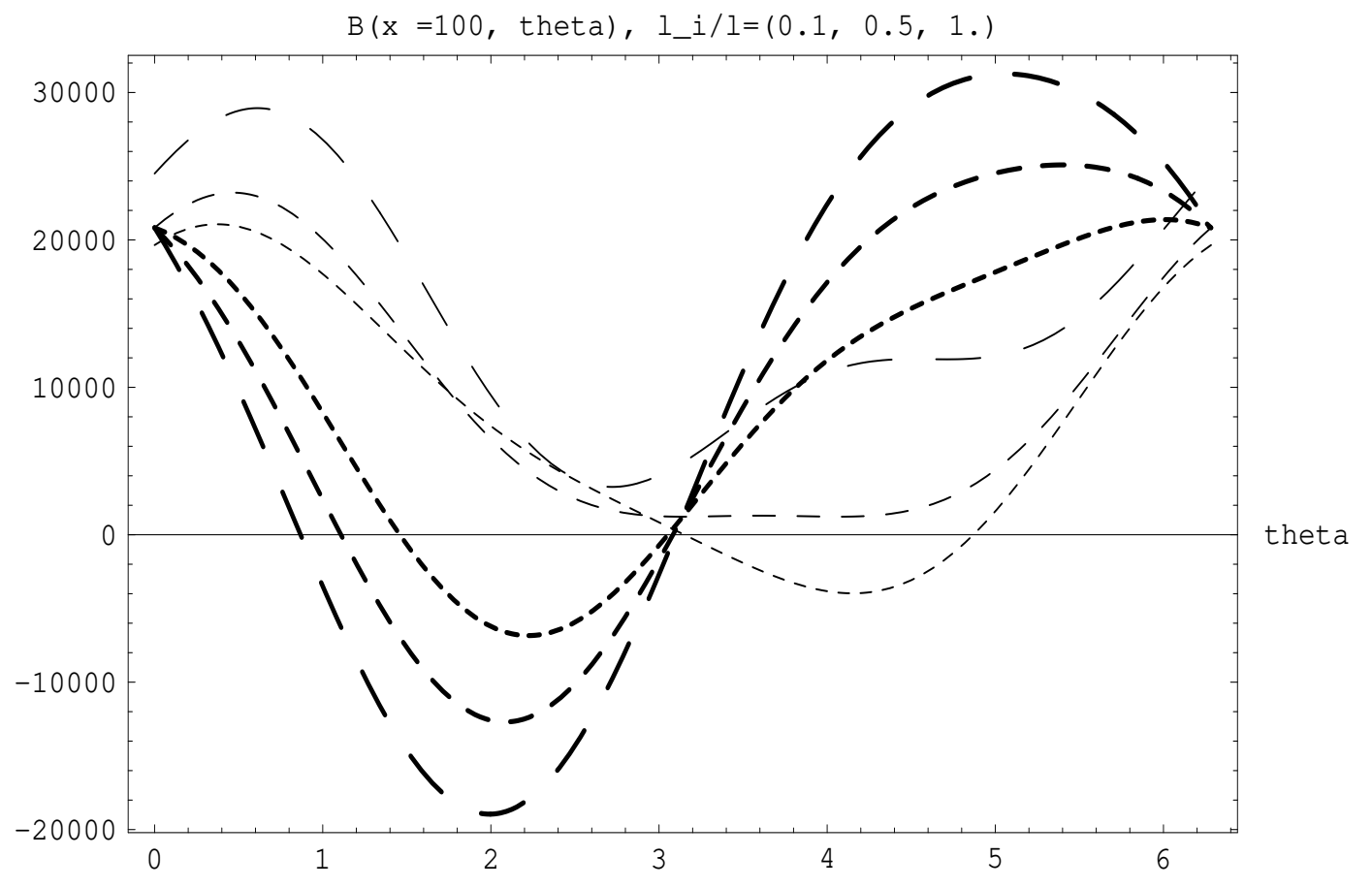

FIG. 4: The coefficient $B(x, \theta)$ for the UFB-2 direction is plotted (in $\mathrm{GeV}^{2}$ units) as a function of $\theta$ for the relevant input parameters set as, $\lambda=0.7, \kappa=0.3, A_{\lambda}=-100 \mathrm{GeV}, m_{H_{d}}^{2}=m_{\tilde{L}, i}^{2}=$ $+100 \mathrm{GeV}^{2}, m_{H_{u}}^{2}=-100 \mathrm{GeV}^{2}, x=100 \mathrm{GeV}$, and a discrete set of choices for $\tilde{\lambda}_{i}$ and $A_{\tilde{\lambda}_{i}}$. The three curves for $A_{\tilde{\lambda}_{i}}=-100 \mathrm{GeV}$ in the three cases $\tilde{\lambda}_{i} / \lambda=(0.1,0.5,1$.$) are drawn in$ light lines with dashes of increasing lengths. The three curves for $\tilde{\lambda}_{i} / \lambda=0.5$ in the three cases $A_{\tilde{\lambda}_{i}}=(100,200,300) \mathrm{GeV}$ are given in thick lines with dashes of increasing lengths. 


$$
\begin{aligned}
& \left.-2 \kappa \tilde{\lambda}_{i} \sqrt{1+w^{2}} x^{2} \sigma^{2}\right)+w^{2} \sigma^{2}\left(2 \tilde{\lambda}_{i}^{2} x^{2}+m_{\tilde{L}_{j}}^{2}+m_{\tilde{E}_{j}^{c}}^{2}\right) \\
& +w^{4} \sigma^{2}\left(\lambda^{2} x^{2}+2 \tilde{\lambda}_{i}^{2} x^{2}+m_{\tilde{L}_{i}}^{2}+m_{H_{u}}^{2}\right)+m_{S}^{2} x^{2}-2 A_{i j j}^{\lambda} \lambda_{i j j} \sigma^{3} w^{3}\left(1+w^{2}\right)^{\frac{1}{2}} \\
& +\sigma^{3} w^{4}\left[2 \lambda_{A j j} \tilde{\lambda}_{A} x+\lambda_{A j j}^{2} \sigma+\lambda_{A i j}^{2} \sigma w^{2}\left(1+w^{2}\right)+\lambda_{i j j}^{2} \sigma\left(1+w^{2}\right)\right] . \quad \text { (IV.12) }
\end{aligned}
$$

Inspection of the above potential indicates that the lepton number violating contributions from $\tilde{\lambda}_{i}$ or $\lambda_{i j k}$ add positive terms to the potential that have the ability to lift the UFB-3 direction. We restrict ourselves to an illustrative example by assigning the NMSSM parameters typical values resulting in a potential with a deep minimum at $\tilde{\lambda}_{i}=0$, and determining whether switching on $\tilde{\lambda}_{i}$ to finite values lifts this minimum. In Fig. 5 we display a representative case in which a deep potential well, produced by choosing negative squared mass values for $m_{H_{u}}^{2}$ and $m_{\tilde{L}_{i}}^{2}$, gets removed upon increasing $\tilde{\lambda}_{i} / \lambda$. We conclude that the lepton number violating interactions can be effective for lifting the UFB-3 field direction.

\section{Electric Charge and Color Breaking Minima}

The lepton number violating interactions may generate a minimum of the scalar potential along the field direction involving finite VEVs for the charged Higgs-slepton fields, $v_{-}=<H_{d}^{-}>, e_{i}=<\tilde{e}_{i}>$. This so-called type II charge breaking minimum has been initially considered by Hirsch et al. [24] for the MSSM with bilinear R-parity violation. We pursue a corresponding analysis for the NMSSM with lepton number violation by solving the equations of motion for the neutral fields in terms of the soft mass parameters $m_{H_{u}}^{2}, m_{H_{d}}^{2}, m_{S}^{2}$ and $m_{\tilde{L}_{i}}^{2}$ (corresponding to prescription II), and substituting these into the two equations of motion for the charged fields, $v_{-}$and $e_{i}$. The equations are displayed in Eq. (A.11) of Appendix A. Note that we can ignore the VEV of the field $\tilde{e}_{i}^{c}$ since $e_{i}^{c}=0$ appears to be the only solution for $\lambda_{i}^{e}=0$. The equations for $v_{-}$and $e_{i}$ depend on the gauge and Yukawa couplings, the soft mass parameters $m_{H_{d} \tilde{L}_{i}}^{2}$, and $v_{i}$.

To start with, we consider the limit of small lepton number violating couplings obtained by expanding the equation of motion in powers of the small parameters $\tilde{\lambda}_{i}$. Substituting the expansion up to leading order in $\tilde{\lambda}_{i}$

$$
v_{d}=v_{d}^{(0)}+\tilde{\lambda}_{i} v_{d}^{(1)}, v_{u}=v_{u}^{0}+\tilde{\lambda}_{i} v_{u}^{(1)}, x=x^{(0)}+\tilde{\lambda}_{i} x^{(1)},
$$




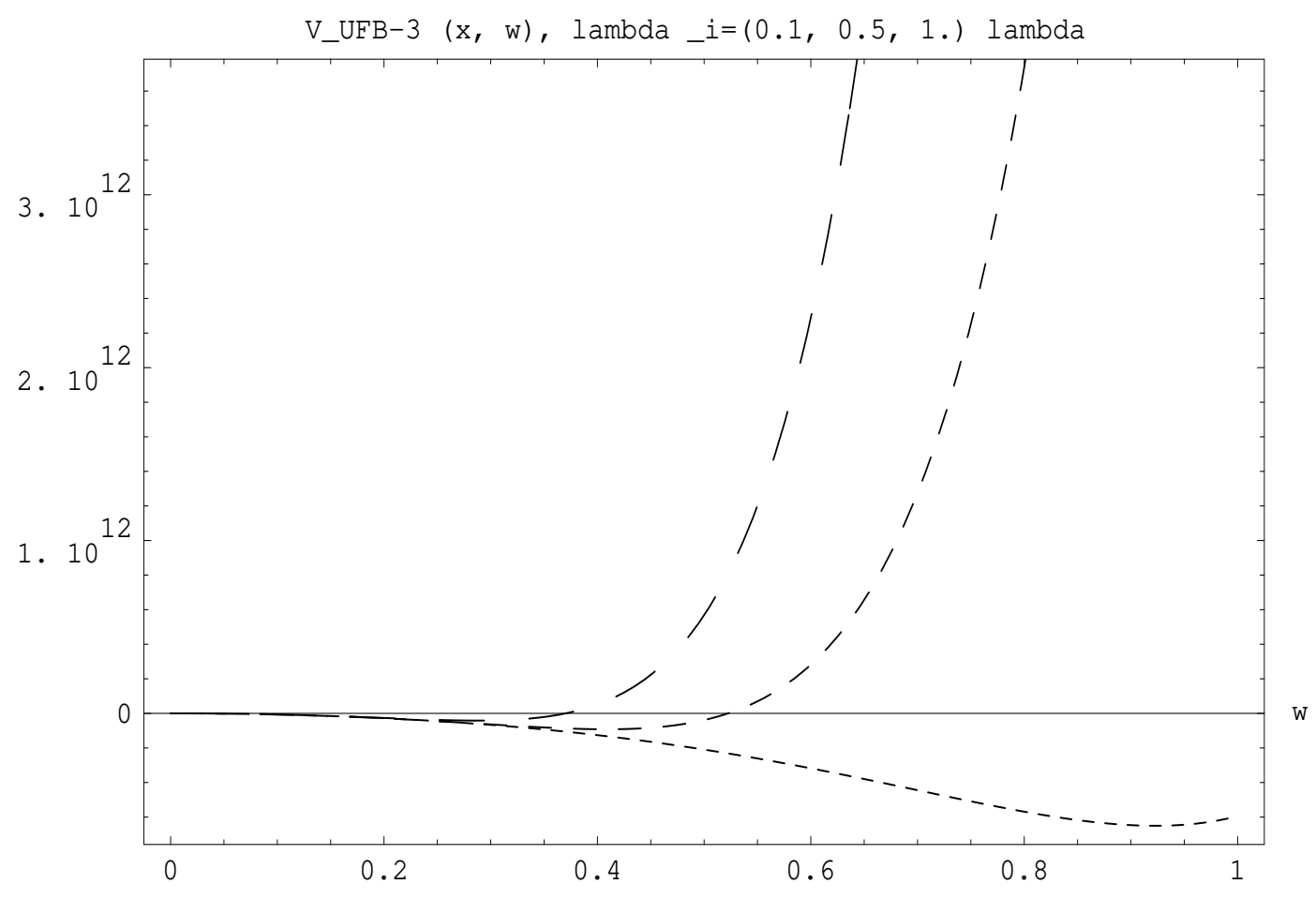

FIG. 5: The potential energy density $V(x, w)$ for the UFB-3 direction is plotted in $\mathrm{GeV}^{4}$ units as a function of $w$ for the choice of input parameters, $\lambda=0.7, \kappa=0.3, A_{\lambda}=500 \mathrm{GeV}, A_{\kappa}=$ $500 \mathrm{GeV}, A_{\tilde{\lambda}_{i}}=500 \mathrm{GeV}, m_{H_{u}}^{2}=m_{H_{d}}^{2}=m_{\tilde{L}, i}^{2}=-200 \mathrm{GeV}^{2}, m_{\tilde{E}_{i}^{c}}^{2}=m_{S}^{2}=100 \mathrm{GeV}^{2}$, with VEV parameters, $x=100 \mathrm{GeV}, \tan \beta=2$ and $\sigma=\lambda x / \lambda_{\tau}^{e}=\lambda x v_{d} / m_{\tau},\left[m_{\tau}=1.777 \mathrm{GeV}\right]$ for the tau-lepton field case. The reduced range of variation of the variable $w$ is explained by the large value assumed by the scaling factor, $\sigma \approx 30.6 x$. The curves for $\tilde{\lambda}_{i} / \lambda=(0.1,0.5,1$.$) are drawn$ with dashes of increasing lengths. 


$$
v_{i}=\tilde{\lambda}_{i} v_{i}^{(1)}, v_{-}=\tilde{\lambda}_{i} v_{-}^{(1)}, e_{i}=\tilde{\lambda}_{i} e_{i}^{(1)},
$$

in the equations of motion for $v_{-}$and $e_{i}$, we find that the equations in leading order of $\tilde{\lambda}_{i}$ only admit the trivial solution $v_{-}=0, e_{i}=0$. Thus, we reach the conclusion that as long as the couplings $\tilde{\lambda}_{i}$ are small compared to unity, the lepton number violating interactions cannot cause the emergence of charge breaking vacuum solution. This conclusion for NMSSM corresponds to that reached in Ref. [24] for the MSSM.

To determine whether a non-trivial solution is favored at finite $\tilde{\lambda}_{i}$, we attempt to solve numerically the equations of motion for $v_{-}$and $e_{i}$, and compare the minimum value $V_{\min }$ with the regular minimum value $V_{M I N}$ at a discrete set of values of the parameters $\tilde{\lambda}_{i}$ and $v_{i}$. For fixed $\tan \beta$, we evaluate $V_{M I N}$ by using the formulas $v_{u}=v^{\prime} \sin \beta, v_{d}=v^{\prime} \cos \beta, \quad\left[v^{\prime}=\right.$ $\left.\left(v^{2}-v_{i}^{2}\right)^{\frac{1}{2}}\right]$. Based on the argument that the singlet VEV $x$ is not strongly affected by the lepton number violating interactions, we identify the value of $x$ in $V_{M I N}$ with its value along the charge breaking field direction in $V_{\min }$. The values of $\tan \beta$ near unity are critical, as nontrivial solutions exist only around $\tan \beta=1$ and disappear quickly at larger values. Since the task of determining the exclusion plot in the $\tilde{\lambda}_{i}, v_{i}$ plane is cumbersome, we use typical values for the relevant parameters, so as to determine to what extent a charge breaking solution at $\lambda_{i}=0$ becomes disallowed by increasing $\lambda_{i}$. We restrict ourselves to solutions with real values of $v_{-}$and $e_{i}$. For the choice of input parameters $\lambda=0.7, g_{1}^{2}=0.127, g_{2}^{2}=$ $0.425, \kappa=0.3, A_{\lambda}=500 \mathrm{GeV}, A_{\tilde{\lambda}_{i}}=500 \mathrm{GeV}, A_{\kappa}=250 \mathrm{GeV}, m_{H_{d} \tilde{L}_{i}}^{2}=100 \mathrm{GeV}^{2}$, with the fixed values of the $\operatorname{VEVs} x=100 \mathrm{GeV}$ and $v_{i}=v / 10$, we find the real solution $\left(v_{-}, e_{i}\right)=(-60.9,585.1) \mathrm{GeV}$ having $V_{\text {min }}-V_{M I N} \approx-8.26 \times 10^{9} \mathrm{GeV}^{4}$ for values of $\tan \beta=1$ and $\tilde{\lambda}_{i} / \lambda=0.1$. However, we find no non-trivial solutions as we increase the coupling $\tilde{\lambda}_{i} / \lambda \in[0.5,1]$. We thus conclude that the lepton number violating interactions have the ability to lift the charge breaking minima.

Finally, we comment briefly on the issue of charge and color breaking minima in the NMSSM by focusing on the field directions [18] described by $v_{u}=\tilde{u}_{i}=\tilde{u}_{i}^{c}=w$ and the singlet field VEV $x$, with all other fields vanishing. Assuming $x$ to be frozen, for simplicity, one finds that the resulting potential,

$$
\left.V_{C C B}=\kappa^{2} x^{4}+m_{S}^{2} x^{2}-\frac{2}{3} A_{\kappa} \kappa x^{3}+w^{2}\left[3 \lambda_{i}^{u 2} w^{2}+2 A_{i}^{u} \lambda_{i}^{u} w+m_{\tilde{U}_{i}}^{2}+m_{\tilde{U}_{i}^{c}}^{2}+m_{H_{u}}^{2}+\sum_{A}(\tilde{\hat{\mathbb{I}}} \not x x)^{2}\right]\right)
$$

does not develop a deep minimum along $w$, for small Yukawa coupling constants $\lambda_{i}^{u}$, provided one satisfies the conditions on the trilinear scalar matter couplings, $A_{i}^{u} \leq$ 
$3\left(m_{H_{u}}^{2}+m_{\tilde{U}_{i}}^{2}+m_{\tilde{U}_{i}^{c}}^{2}+\left(\tilde{\lambda}_{A} x\right)^{2}\right)^{\frac{1}{2}}$. This approximate result suggests that the bounds on $A_{i}^{u}$ should become weaker upon increasing $\tilde{\lambda}_{i}$. The general field direction described by [22] $\left(\left|v_{u}\right|,\left|u_{i}\right|,\left|u_{i}^{c}\right|,\left|v_{d}\right|,\left|v_{j}\right|\right)=\left(1, \alpha, \beta, \gamma, \gamma_{L}\right) w$ along with the singlet field VEV $x$, involves a more elaborate discussion which we shall not pursue here.

\section{CONCLUSIONS}

In the present work we have examined the effect of lepton number conserving and violating Yukawa couplings of same size, $\tilde{\lambda}_{i} \approx \lambda$, on NMSSM. One expects significant modifications for the scalar sector observables since the spontaneous electroweak gauge symmetry breaking is now linked to both the down type Higgs boson and sleptons. An important challenge was raised by the need to define a simple parameterization of the model consistent with the constraints on the light neutrinos without specifying in detail the underlying dynamics. Noting that the dominant contributions to the neutrino Majorana mass matrix are controlled by the effective alignment parameters $\tilde{\lambda}_{i}^{\prime}=\tilde{\lambda}_{i}-\lambda v_{i} / v_{d}$ and $\eta_{i}=\frac{A_{\tilde{\lambda}_{i}} \tilde{\lambda}_{i}}{A_{\lambda} \lambda}-\frac{v_{i}}{v_{d}}$, we proceeded by treating $\tilde{\lambda}_{i}$ as free parameters while determining the values of the sneutrino VEVs $v_{i}$ and $A_{\tilde{\lambda}_{i}}$ through the restrictive conditions, $\tilde{\lambda}_{i}^{\prime} \simeq 0, \eta_{i} \simeq 0$. There is no unique prescription, and the one used may well single out a non-generic region of the NMSSM parameter space. To answer this objection, one could attempt building a supergravity unified model with a $U(1)_{R}$ symmetry spontaneously broken in a hidden sector so as to check whether this respects an approximate dynamical alignment robust under the renormalization group scale evolution, following a similar analysis as that of Nilles and Polonsky [27].

In the first part of the present work we examined the impact of the lepton number violating interactions on the mass spectra of scalars. The vacuum stability constraints were found to restrict the VEV parameters, $\tan \beta=v_{u} / v_{d}$ and $x$, to narrower intervals than in the lepton number conserving case. This property is reflected in the fact that the bounds

on $\tilde{\lambda}_{i}$ become stronger for larger $\tan \beta$ and $x$. There are certain analogies between our study and that developed for the MSSM with bilinear R-parity violation by Davidson et al. [14] in terms of the basis invariant parameter $\delta_{R}$, corresponding to $\sum_{i} \eta_{i}$ in our basis choice, at fixed values of the CP-odd scalar masses. The conclusions in the latter work regarding the reduced range of variation of $\tan \beta$ and the size of the corrections to the scalar sector masses are qualitatively similar to ours. However, no meaningful comparison can be made because 
of the different parameterizations.

The second part of the present work was devoted to a qualitative study of the vacuum stability contraints from the UFB field directions and the CCB minima. The discussion for this case, unlike that in the first part, does not bear directly on the implementation of the parameter alignment conditions. Based on illustrative examples covering a small part of the parameter space, we found that the lepton number violating interactions may have a positive impact on the regular vacuum stability provided the coupling constants $\tilde{\lambda}_{i}$ and $A_{\tilde{\lambda}_{i}}$ assume large enough values. Since the approach of selecting field directions based on the renormalization group cannot be developed in the NMSSM by analytic means only, its advantage over a systematic numerical exploration covering the full field space is not clear. Nevertheless, our discussion indicates that pursuing the renormalization group approach on more quantitative grounds is worthwhile. The recent progress in developing efficient numerical methods to search the global minimum of the scalar potential in multidimensional field spaces [45] could be useful for further studies along these lines.

\section{APPENDIX A: USEFUL FORMULAS AND CONVENTIONS}

The $R$-parity conserving and $R$-parity violating parts of the NMSSM superpotential can be written as

$$
\begin{aligned}
& W_{R P C}=\lambda_{j k}^{u} H_{u} Q_{j} U_{k}^{c}+\lambda_{j k}^{d} H_{d} Q_{j} D_{k}^{c}+\lambda_{j k}^{e} H_{d} L_{j} E_{k}^{c}+\lambda H_{d} H_{u} S-\frac{\kappa}{3} S^{3}, \\
& W_{R P V}=\frac{1}{2} \lambda_{i j k} L_{i} L_{j} E_{k}^{c}+\lambda_{i j k}^{\prime} L_{i} Q_{j} D_{k}^{c}+\frac{1}{2} \lambda_{i j k}^{\prime \prime} U_{i}^{c} D_{j}^{c} D_{k}^{c}+\tilde{\lambda}_{i} L_{i} H_{u} S .
\end{aligned}
$$

The covariant four vector notation for the lepton and the down-type Higgs superfields, and for the Yukawa couplings, employed in this paper is

$$
\begin{aligned}
& L_{A}=\left(H_{d}, L_{i}\right)=\left(\left(\begin{array}{c}
H_{d}^{0}, \tilde{H}_{d}^{0} \\
H_{d}^{-}, \tilde{H}_{d}^{-}
\end{array}\right),\left(\begin{array}{c}
\tilde{\nu}_{i}, \nu_{i} \\
\tilde{e}_{i}, e_{i}
\end{array}\right)\right), \\
& \tilde{\lambda}_{A}=\left(\lambda, \tilde{\lambda}_{i}\right), \lambda_{A j k}=\left(\lambda_{j k}^{e}, \lambda_{i j k}\right), \lambda_{A j k}^{\prime}=\left(\lambda_{j k}^{d}, \lambda_{i j k}^{\prime}\right) .
\end{aligned}
$$

We use the following convention in writing the multiplication of chiral electroweak doublet superfields:

$$
\begin{aligned}
L_{A} H_{u} & \equiv L_{A} \cdot \epsilon \cdot H_{u}=\nu_{A} H_{u}^{0}-e_{A} H_{u}^{+}=\left(H_{d}^{0} H_{u}^{0}-H_{d}^{-} H_{u}^{+}\right)+\left(\nu_{i} H_{u}^{0}-e_{i} H_{u}^{+}\right), \\
H_{u} Q & \equiv H_{u} \cdot \epsilon \cdot Q=H_{u}^{+} D-H_{u}^{0} U, H_{d} Q \equiv H_{d} \cdot \epsilon \cdot Q=H_{d}^{0} D-H_{d}^{-} U,
\end{aligned}
$$


where $\epsilon$ is the $2 \times 2$ antisymmetric matrix with $\epsilon_{12}=-\epsilon_{21}=1$. The $R$-parity conserving and $R$-parity violating contributions to the trilinear part of the potential can be written as

$$
\begin{aligned}
V_{R P C}^{s o f t} & =-A_{j k}^{u} \lambda_{j k}^{u} H_{u} \tilde{Q}_{j} \tilde{U}_{k}^{c}-A_{j k}^{d} \lambda_{j k}^{d} H_{d} \tilde{Q}_{D_{k}}^{c}-A_{j k}^{e} \lambda_{j k}^{e} H_{d} \tilde{L}_{j} \tilde{E}_{k}^{c}-A_{\lambda} \lambda H_{d} H_{u} S-\frac{A_{\kappa} \kappa}{3} S^{3}, \\
V_{R P V}^{s o f t} & =-\frac{1}{2} A_{i j k}^{\lambda} \lambda_{i j k} \tilde{L}_{i} \tilde{L}_{j} \tilde{E}_{k}^{c}-A_{i j k}^{\lambda^{\prime}} \lambda_{i j k}^{\prime} \tilde{L}_{i} \tilde{Q}_{j} \tilde{D}_{k}^{c}-\frac{1}{2} A_{i j k}^{\lambda^{\prime \prime}} \lambda_{i j k}^{\prime \prime} \tilde{U}_{i}^{c} \tilde{D}_{j}^{c} \tilde{D}_{k}^{c}-A_{\tilde{\lambda}_{i}} \tilde{\lambda}_{i} \tilde{L}_{i} H_{u} S+H(\text { Ac7. })
\end{aligned}
$$

The relationship that we adopted between the superpotential and the effective Lagrangian uses the convention $L_{E F F}=+[W]_{F}+H . c .=-\left|W_{i}\right|^{2}+\frac{1}{2} W_{i j} \psi_{i} \psi_{j}+$ H.c. in contrast to certain authors which use the opposite sign convention, $L_{E F F}=-\left([W]_{F}+H . c.\right)=-\left|W_{i}\right|^{2}-$ $\frac{1}{2} W_{i j} \psi_{i} \psi_{j}+H . c$. . The latter sign convention is that adopted, for instance, in the studies of the NMSSM by Miller et al., [31] and by Barger et al., [32]. Accounting for this fact, we obtain the following correspondence between our notations and that of the latter authors: $\lambda \rightarrow h_{s}, A_{\lambda} \rightarrow A_{s}, \kappa \rightarrow \kappa, A_{\kappa} \rightarrow-A_{\kappa}, \lambda^{u} \rightarrow h_{t}, A_{t}^{u} \rightarrow A_{t}, x \rightarrow s / \sqrt{2}$. We have compared our formulas for the scalar sector potential and mass matrices and for the neutralino sector and found complete agreement.

The scalar potential for the electrically neutral fields, with the electrically charged and color non-singlet scalar fields set to zero, is given by

$$
\begin{aligned}
V_{F}+V_{D}+V_{\text {soft }} & =\left|\lambda v_{d}+\tilde{\lambda}_{i} v_{i}\right|^{2}|x|^{2}+\left(\left|\lambda v_{u}\right|^{2}+\left|\tilde{\lambda}_{i} v_{i}\right|^{2}\right)|x|^{2} \\
& +\left|v_{u}\left(\lambda v_{d}+\tilde{\lambda}_{i} v_{i}\right)-\kappa x^{2}\right|^{2}+G_{+}^{2}\left(\left|v_{u}\right|^{2}-\left|v_{d}\right|^{2}-\left|v_{i}\right|^{2}\right)^{2} \\
& +\left[-A_{\lambda} \lambda v_{d} v_{u} x-A_{\tilde{\lambda}_{i}} \tilde{\lambda}_{i} v_{i} v_{u} x-\frac{A_{\kappa} \kappa}{3}|x|^{3}+\mu_{A u}^{2} v_{A} v_{u}+m_{H_{d} \tilde{L}_{i}}^{2} v_{d} v_{i}^{\star}+\text { H. c. }\right] \\
& +m_{H_{u}}^{2}\left|v_{u}\right|^{2}+m_{H_{d}}^{2}\left|v_{d}\right|^{2}+m_{\tilde{L}_{i}}^{2}\left|v_{i}\right|^{2}+m_{S}^{2}|x|^{2} .
\end{aligned}
$$

The relevant neutral and charged field VEVs are denoted as, $\left\langle H_{u}^{0}\right\rangle=v_{u},\left\langle H_{d}^{0}\right\rangle=$ $v_{d},<S>=x,<\tilde{\nu}_{i}>=v_{i}$, and $<H_{d}^{-}>=v_{-},<\tilde{e}_{i}>=e_{i},<\tilde{e}_{i}^{c}>=e_{i}^{c},<\tilde{q}_{i}>=q_{i},<$ $\tilde{q}_{i}^{c}>=q_{i}^{c}, \quad\left[\tilde{q}_{i}=\left(\tilde{u}_{i}, \tilde{d}_{i}\right), \tilde{q}_{i}^{c}=\left(\tilde{u}_{i}^{c}, \tilde{d}_{i}^{c}\right)\right]$. Our choice of field basis, $v_{+}=0$, obviates the need to consider the electrically charged direction $\left\langle H_{u}^{+}\right\rangle=v^{+}$. The formulas determining the soft masses of the Higgs bosons and sleptons through the minimization equations of the neutral fields in prescription I (using $m_{\tilde{L}_{i}}^{2}$ as free parameters) for finite values of the neutral and charged fields are given by

$$
\begin{aligned}
& m_{H_{u}}^{2}=\frac{g_{1}^{2}}{4}\left(e_{i}^{2}+v_{-}^{2}+v_{d}^{2}-v_{u}^{2}\right)+\frac{g_{2}^{2}}{4}\left(-e_{i}^{2}-v_{-}^{2}+v_{d}^{2}-v_{u}^{2}\right)-\lambda^{2} v_{d}^{2} \\
& +\frac{A_{\lambda} \lambda v_{d} x}{v_{u}}-\lambda^{2} x^{2}-\tilde{\lambda}_{i}^{2} x^{2}+\frac{\kappa \lambda v_{d} x^{2}}{v_{u}}
\end{aligned}
$$




$$
\begin{aligned}
& +\left(-2 \lambda \tilde{\lambda}_{i} v_{d}+\frac{A_{\tilde{\lambda}_{i}} \tilde{\lambda}_{i} x}{v_{u}}+\frac{\kappa \tilde{\lambda}_{i} x^{2}}{v_{u}}\right) v_{i}+\left(\frac{g_{1}^{2}}{4}+\frac{g_{2}^{2}}{4}-\tilde{\lambda}_{i}^{2}\right) v_{i}^{2}, \\
& m_{H_{d}}^{2}=\frac{g_{1}^{2}}{4}\left(-e_{i}^{2}-v_{-}^{2}-v_{d}^{2}+v_{u}^{2}\right)+\frac{g_{2}^{2}}{4}\left(e_{i}^{2}-v_{-}^{2}-v_{d}^{2}+v_{u}^{2}\right) \\
& -\lambda^{2} v_{u}{ }^{2}+\frac{A_{\lambda} \lambda v_{u} x}{v_{d}}-\lambda^{2} x^{2}+\frac{\kappa \lambda v_{u} x^{2}}{v_{d}}+\frac{1}{v_{d}^{2}}\left[\left(-A_{\tilde{\lambda}_{i}} \tilde{\lambda}_{i} v_{u} x-\kappa \tilde{\lambda}_{i} v_{u} x^{2}\right) v_{i}\right. \\
& +\left(\frac{g_{1}^{2}}{4}\left(e_{i}^{2}+v_{-}^{2}-v_{u}^{2} \frac{g_{2}^{2}}{4}\left(e_{i}^{2}-v_{-}^{2}-v_{u}^{2}\right)+m_{\tilde{L}_{i}}^{2}+\tilde{\lambda}_{i}^{2}\left(v_{u}^{2}+x^{2}\right)\right) v_{i}^{2}+\frac{g_{1}^{2}+g_{2}^{2}}{4} v_{i}^{4}\right] \\
& m_{S}^{2}=-v_{-}{ }^{2} \lambda^{2}-2 e_{i} v_{-} \lambda \tilde{\lambda}_{i}-e_{i}{ }^{2} \tilde{\lambda}_{i}^{2}-\lambda^{2} v_{d}^{2}+2 \kappa \lambda v_{d} v_{u}-\lambda^{2} v_{u}{ }^{2} \\
& -\tilde{\lambda}_{i}^{2} v_{u}{ }^{2}+\frac{A_{\lambda} \lambda v_{d} v_{u}}{x}+A_{\kappa} \kappa x-2 \kappa^{2} x^{2}+\left(-2 \lambda \tilde{\lambda}_{i} v_{d}+2 \kappa \tilde{\lambda}_{i} v_{u}+\frac{A_{\tilde{\lambda}_{i}} v_{u}}{x}\right) v_{i}-\tilde{\lambda}_{i}^{2} v_{i}^{2}, \\
& m_{H_{d} \tilde{L}_{i}}^{2}=-\frac{1}{2} g_{2}^{2} e_{i} v_{-}-\lambda \tilde{\lambda}_{i}\left(v_{u}^{2}+x^{2}\right)+\frac{1}{v_{d}}\left[\tilde{\lambda}_{i} v_{u} x\left(A_{\tilde{\lambda}_{i}}+\kappa x\right)\right. \\
& \left.+\left(\frac{g_{1}^{2}}{4}\left(-e_{i}^{2}-v_{-}^{2}-v_{d}^{2}+v_{u}^{2}\right)+\frac{g_{2}^{2}}{4}\left(-e_{i}^{2}+v_{-}^{2}-v_{d}^{2}+v_{u}^{2}\right)-m_{\tilde{L}_{i}}^{2}\right) v_{i}-\frac{g_{1}^{2}+g_{2}^{2}}{4} v_{i}^{3}\right] . \quad \text { (A.9) }
\end{aligned}
$$

The equations of motion for the $L_{A}=\left(H_{d}, L_{i}\right)$ fields can be expressed in the $S U(4)$ group covariant notation as

$$
\begin{aligned}
& \hat{M}_{\tilde{L}, A B}^{2} v_{B}=\tilde{\lambda}_{A} x v_{u}\left(A_{\tilde{\lambda}_{A}}+\kappa x\right), \\
& {\left[\hat{M}_{\tilde{L}, A B}^{2}=m_{\tilde{L}, A B}^{2}+\tilde{\lambda}_{A} \tilde{\lambda}_{B}\left(v_{u}^{2}+x^{2}\right)+2 G_{+}^{2}\left(\hat{v}_{d}^{2}-v_{u}^{2}\right) \delta_{A B}\right] .}
\end{aligned}
$$

The above structure of the neutral scalars squared mass matrix satisfies the important property that the alignment $\tilde{\lambda}_{A} \propto v_{A}$ is satisfied if and only if $\tilde{\lambda}_{A}$ is an eigenvalue of the matrix $\hat{M}_{\tilde{L}, A B}^{2}$. This result generalizes that found in the MSSM with R-parity violation [10, $46]$.

The minimization equations for the charged slepton fields in prescription II (using $m_{H_{d} \tilde{L}_{i}}^{2}$ as free parameters) are given by

$$
\begin{aligned}
& e_{i}^{c}: 0=2 e_{i}^{c 3} g_{1}^{2}+e_{i}^{c}-\left[e_{i}^{2} g_{1}^{2}+2 m_{\tilde{E}^{c}}^{2}-g_{1}^{2}\left(v_{-}^{2}+v_{i}^{2}+v_{d}^{2}-v_{u}{ }^{2}\right)\right]-2 A_{\tau}^{e} \lambda_{\tau}^{e} e_{i} v_{d}, \\
& v_{-}: 0=\frac{1}{v_{d}}\left[e_{i}^{2} g_{2}^{2} v_{-} v_{d}+v_{-} v_{u}\left(g_{2}^{2} v_{d} v_{u}-2 \lambda^{2} v_{d} v_{u}+2 A_{\lambda} \lambda x+2 \kappa \lambda x^{2}\right)\right. \\
& \left.+2 e_{i}\left(m_{H_{d} \tilde{L}_{i}}^{2} v_{d}+\lambda \tilde{\lambda}_{i} v_{d} x^{2}\right)\right] \\
& +\frac{1}{v_{d}}\left[\left(e_{i} g_{2}^{2}\left(-v_{-}^{2}+v_{d}^{2}\right)-2 v_{-}\left(m_{H_{d} \tilde{L}_{i}}^{2}+\lambda \tilde{\lambda}_{i}\left(v_{u}{ }^{2}+x^{2}\right)\right)\right) v_{i}\right]-g_{2}^{2} v_{-} v_{i}^{2}, \\
& e_{i}: 0=-\frac{e_{i}}{v_{i}}\left(e_{i} g_{2}^{2} v_{-} v_{d}+2\left(m_{H_{d} \tilde{L}_{i}}^{2} v_{d}+\lambda \tilde{\lambda}_{i} v_{d} v_{u}{ }^{2}-A_{\tilde{\lambda}_{i}} \tilde{\lambda}_{i} v_{u} x+\lambda \tilde{\lambda}_{i} v_{d} x^{2}-\kappa \tilde{\lambda}_{i} v_{u} x^{2}\right)\right)
\end{aligned}
$$




$$
+e_{i}\left(-2 \tilde{\lambda}_{i}^{2} v_{u}{ }^{2}+g_{2}^{2}\left(v_{-}^{2}-v_{d}^{2}+v_{u}{ }^{2}\right)\right)+2 v_{-}\left(m_{H_{d} \tilde{L}_{i}}^{2}+\lambda \tilde{\lambda}_{i} x^{2}\right)+g_{2}^{2} v_{-} v_{d} v_{i}
$$

[1] See, e. g., A. Y. Smirnov, [arXiv:hep-ph/0702061].

[2] P. Minkowski, Phys. Lett. B 67, 421 (1977); M. Gell-Mann, P. Ramond and R. Slansky, In Supergravity, eds. P. van Nieuwenhuizen and D. Z. Freedman (North-Holland, Amsterdam, 1979), p. 315; T. Yanagida, In Proceedings of the Workshop on the Baryon Number of the Universe and Unified Theories, Tsukuba, Japan, 13-14 Feb 1979; R. N. Mohapatra and G. Senjanovic, Phys. Rev. Lett. 44, 912 (1980).

[3] See, e. g., J. C. Romao, [arXiv:hep-ph/0510411].

[4] M. Chemtob and P. N. Pandita, Phys. Rev. D 73, 055012 (2006) [arXiv:hep-ph/0601159].

[5] P. N. Pandita and P. F. Paulraj, Phys. Lett. B 462, 294 (1999) [arXiv:hep-ph/9907561].

[6] P. N. Pandita, Phys. Rev. D 64, 056002 (2001) [arXiv:hep-ph/0103005].

[7] R. Dermisek and J. F. Gunion, Phys. Rev. Lett. 95, 041801 (2005) [arXiv:hep-ph/0502105].

[8] Y. Grossman and H. E. Haber, Phys. Rev. D 59, 093008 (1999) [arXiv:hep-ph/9810536].

[9] Y. Grossman and H.E. Haber, Phys. Rev. Lett. 78, 3438 (1997).

[10] Y. Grossman and H.E. Haber, Phys. Rev. D63, 075011 (2001) [arXiv:hep-ph/0005276].

[11] Y. Grossman and H.E. Haber Phys. Rev. D67, 036002 (2003) [arXiv:hep-ph/0210273],

[12] S. Davidson and M. Losada, JHEP 0005, 021 (2000) [arXiv:hep-ph/0005080].

[13] S. Davidson and M. Losada, Phys. Rev. D 65, 075025 (2002) [arXiv:hep-ph/0010325].

[14] S. Davidson, M. Losada, and N. Rius, Nucl. Phys. B587, 118 (2000) [arXiv:hep-ph/9911317]

[15] A. Abada, S. Davidson and M. Losada, Phys. Rev. D65, 075010 (2002) [arXiv:hepph/0111332]; A. Abada, G. Bhattacharyya, and M. Losada, Phys. Rev. D66, 071701(R) (2002) [arXiv:hep-ph/0208009]; A. Abada and M. Losada, Nucl. Phys. B585, 45 (2000); Phys. Lett. B492, 310 (2000).

[16] A. Abada, G. Bhattacharyya, and G. Moreau, Phys. Lett. B642, 503 (2006) [arXiv:hep$\mathrm{ph} / 0606179]$.

[17] A. Abada and G. Moreau, JHEP 0608, 044 (2006) [arXiv:hep-ph/0604216].

[18] J. M. Frere, D. R. T. Jones and S. Raby, Nucl. Phys. B 222, 11 (1983).

[19] M. Claudson, L. J. Hall and I. Hinchliffe, Nucl. Phys. B 228, 501 (1983). 
[20] H. P. Nilles, M. Srednicki and D. Wyler, Phys. Lett. B 124, 337 (1983).

[21] H. Komatsu, Phys. Lett. B 215, 323 (1988).

[22] J. A. Casas, arXiv:hep-ph/9707475. J. A. Casas, A. Lleyda and C. Munoz, Phys. Lett. B 389, 305 (1996) [arXiv:hep-ph/9606212]; J. A. Casas, A. Lleyda and C. Munoz, Nucl. Phys. B 471, 3 (1996) [arXiv:hep-ph/9507294].

[23] S. A. Abel and C. A. Savoy, Nucl. Phys. B 532, 3 (1998) [arXiv:hep-ph/9803218].

[24] M. Hirsch, C. Hugonie, J. C. Romao and J. W. F. Valle, JHEP 0503, 020 (2005) [arXiv:hep$\mathrm{ph} / 0411129]$.

[25] T. Banks, Y. Grossman, E. Nardi, and Y. Nir, Phys. Rev. D52, 5319 (1995) [arXiv:hep$\mathrm{ph} / 9505248]$.

[26] F. Borzumati, Y. Grossman, E. Nardi, and Y. Nir, Phys. Lett. B384, 123 (1996) [arXiv:hepph/9606251]; Y. Grossman, Y. Nir and Y. Shadmi, JHEP 9810:007,1998 [arXiv:hep$\mathrm{ph} / 9808355]$.

[27] H.-P. Nilles and N. Polonsky, Nucl. Phys. B478, 3 (1996).

[28] R. Hempfling, Nucl. Phys. B484, 33 (1997).

[29] P. N. Pandita, Phys. Lett. B 318, 338 (1993).

[30] P. N. Pandita, Z. Phys. C 59 (1993) 575.

[31] D.J. Miller, R. Nevzorov, and P.M. Zerwas, Nucl. Phys. B681, 3 (2004) [arXiv:hep$\mathrm{ph} / 0304049]$.

[32] V. Barger, P. Langacker, H. S. Lee and G. Shaughnessy, [arXiv:hep-ph/0603247]; V. Barger, P. Langacker and H. S. Lee, Phys. Lett. B 630, 85 (2005) [arXiv:hep-ph/0508027].

[33] J. R. Ellis, J. F. Gunion, H. E. Haber, L. Roszkowski and F. Zwirner, Phys. Rev. D 39, 844 (1989).

[34] T. Hambye, E. Ma, and U. Sarkar, Phys. Rev. D62, 015010 (2000) [arXiv:hep-ph/9911422]; Nucl. Phys. B590, 429 (2000) [arXiv:hep-ph/0006173].

[35] E.J. Chun, D.-W. Jung, and J.D. Park, [arXiv:hep-ph/0211310].

[36] J.P. Derendinger and C. Savoy, Nucl. Phys. B237, 307 (1984).

[37] L.E. Ibanez and J. Mas, Nucl. Phys. B286, 107 (1987).

[38] J.F. Gunion, H.E. Haber, and M. Sher, Nucl. Phys. B306, 1 (1988).

[39] R. Nevrozov and M.A. Trusov, [arXiv:hep-ph/0110363].

[40] R.B. Nevzorov and M.A.Trusov, Phys.Atom.Nucl. 65 (2002) 335-344; Yad.Fiz. 65 (2002) 
359-368 [arXiv:hep-ph/0301179]; ibidem, Phys.Atom.Nucl. 64 (2001) 1513-1530; Yad.Fiz. 64 (2001) 1589-1606 [arXiv:hep-ph/0112301]; R.B. Nevzorov, K.A. Ter-Martirosyan, and M. A. Trusov, [arXiv:hep-ph/0301068].

[41] U. Ellwanger, M.R. de Traubenberg, and C. Savoy, Z. Phys. C67, 665 (1995).

[42] S.F. King and P. L. White, Phys. Rev. D53, 4049 (1996).

[43] J.F. Gunion, H.E. Haber, T. Moroi, [arXiv:hep-ph/9610337].

[44] U. Ellwanger, J.F. Gunion, and C. Hugonie, JHEP C0507, 04 (2005) [arXiv:hep-ph/0503203].

[45] M. Maniatis, A. von Manteuffel, and O. Nachtmann, Eur. Phys. J. C49: 1067-1076, 2007 [arXiv:hep-ph/0608314].

[46] Y. Grossman and S. Rakshit, Phys. Rev. D69, 093002 (2004). 